\title{
COMPOSITION OPERATORS IN THE DIRICHLET SERIES SETTING
}

\author{
HERVÉ QUEFFÉLEC \\ U.F.R. de Mathématiques, Université de Lille 1 \\ 59655 Villeneuve d'Ascq Cedex, France \\ E-mail: Herve.Queffelec@math.univ-lille1.fr
}

\begin{abstract}
In this work, we begin with a survey of composition operators on the Hardy space $H^{2}$ and on the Wiener algebra $A^{+}$of absolutely convergent Taylor series, with special emphasis on their compactness, or invertibility, or isometric character. The main results are due respectively to J. Shapiro and D. Newman.

In a second part, we present more recent results, due to Gordon and Hedenmalm on the one hand, and to Bayart, the author et al. on the other hand, concerning the analogues of $H^{2}$ and $A^{+}$in the setting of Dirichlet series. We are led to the intermediate study of Taylor series in several, or countably many, variables. We finish with some open problems.
\end{abstract}

0. Introduction. The context in which composition operators are generally studied is the following: $\Omega$ is an open set of $\mathbb{C}$ (occasionally of $\mathbb{C}^{d}$ ), $H(\Omega)$ is the set of holomorphic functions $f: \Omega \rightarrow \mathbb{C}$, endowed with its natural topology of compact convergence, $X$ is a Banach space continuously embedded in $H(\Omega)$, and $\phi$ is a holomorphic self-map of $\Omega$ (in short $\phi \in H(\Omega, \Omega))$. The composition operator $C_{\phi}$ with symbol $\phi$ is then formally defined by $C_{\phi}(f)=f \circ \phi$, and maps in particular $X$ to $H(\Omega)$. And the question is: when does $C_{\phi}$ map $X$ to itself? (Then, $C_{\phi}$ is automatically bounded, by the closed graph Theorem). The answer depends very much on the space $X$ and on the map $\phi$, and a big amount of research work has been devoted to this question, in the last twenty years, with special emphasis on the connections between the operator-theoretic properties of $C_{\phi}: X \rightarrow X$, and the analytic properties of $\phi: \Omega \rightarrow \Omega$. We refer to the books [S2], [CoMcCl] and to the articles which they quote; the case of Hardy or of Bergman spaces is studied there in great detail. We can also have two different Banach spaces $X$ and $Y \subset H(\Omega)$, and study the $C_{\phi}$ 's mapping $X$ to $Y$; see the interesting paper [HJ] in this direction.

In this rather short survey, we shall deliberately restrict ourselves to four spaces $X$ and to four theoretical aspects of the operator $C_{\phi}$, namely:

2000 Mathematics Subject Classification: Primary 47B33; Secondary 30B50, 42B35.

Key words and phrases: composition operators, Taylor series, Dirichlet series.

The paper is in final form and no version of it will be published elsewhere. 
1) Boundedness;

2) Compactness;

3) Automorphic character;

4) Isometric character.

Other important theoretical aspects, like spectrum, hypercyclicity, etc. will be left aside.

Our four spaces will be two Hilbert spaces and two Banach algebras:

1) The Hardy-Hilbert space $H^{2}$ of analytic functions $f(z)=\sum_{0}^{\infty} a_{n} z^{n}$ with squaresummable coefficients: $\|f\|^{2}=\sum_{0}^{\infty}\left|a_{n}\right|^{2}<\infty$. We often write $\hat{f}(n)$ instead of $a_{n}$.

2) The Wiener-Banach algebra $A^{+}$of analytic functions $f(z)=\sum_{0}^{\infty} a_{n} z^{n}$ with summable coefficients: $\|f\|=\sum_{0}^{\infty}\left|a_{n}\right|<\infty$.

3) The Hardy-Dirichlet Hilbert space $\mathcal{H}^{2}$ of analytic functions $f$ admitting a Dirichlet series expansion $f(s)=\sum_{1}^{\infty} a_{n} n^{-s}$ with square-summable coefficients: $\|f\|^{2}=$ $\sum_{1}^{\infty}\left|a_{n}\right|^{2}<\infty$.

4) The Wiener-Dirichlet algebra $\mathcal{A}^{+}$of analytic functions admitting a Dirichlet series expansion $f(s)=\sum_{1}^{\infty} a_{n} n^{-s}$ with summable coefficients: $\|f\|=\sum_{1}^{\infty}\left|a_{n}\right|<\infty$.

As we shall see, the functional properties of $H^{2}$ and $\mathcal{H}^{2}$, or of $A^{+}$and $\mathcal{A}^{+}$, are quite different; in particular, to study this last space, we will be led to the intermediate study of the spaces $A^{+}\left(\mathbb{T}^{k}\right), A^{+}\left(\mathbb{T}^{\infty}\right)$, of absolutely convergent Taylor series in $k$ (resp. infinitely many) complex variables.

Two general and easy properties will be frequently used:

$$
\begin{gathered}
C_{\phi_{1} \circ \phi_{2}}=C_{\phi_{2}} \circ C_{\phi_{1}} \quad \text { for any } \phi_{1}, \phi_{2}: \Omega \rightarrow \Omega . \\
C_{\phi}^{*}\left(K_{a}\right)=K_{\phi(a)} \quad \text { for any } a \in \Omega .
\end{gathered}
$$

(Here, $X$ is a Hilbert space of analytic functions on $\Omega$, with reproducing kernel $K$, and $C_{\phi}^{*}$ is the adjoint of $C_{\phi}$ ).

A word on the notations and on the content: when proofs are given in full detail in existing papers, we generally omit, or briefly sketch, them. Conversely, when they are only sketched in the literature, we add some detail, for the convenience of the reader.

$D$ will denote the open unit disk; we will frequently write the complex number $s$ with Riemann's notation:

$$
s=\sigma+i t, \quad \sigma=\Re s, \quad t=\Im s .
$$

$\mathbb{C}_{\theta}$ will denote the open half-plane $\Re s>\theta$, where $\theta$ is a given real number.

The paper is divided into six sections: in Section 1, we study the Hardy space $H^{2}$; in Section 2, we study the Wiener algebra $A^{+}$; Section 3 introduces to the Dirichlet series setting; Sections 4 and 5 study the Dirichlet analogues $\mathcal{H}^{2}, \mathcal{A}^{+}$of $H^{2}$ and $A^{+}$; finally, Section 6 is devoted to some concluding remarks and questions.

1. The Hardy spaces $H^{2}$. Let us first fix some notation, and recall some basic facts: $\phi$ will always denote an analytic self-map of $D ; m$ will denote the Haar measure of the circle $\mathbb{T}$, and we shall write $\int \ldots d m$ for $\int_{0}^{2 \pi} \ldots \frac{d \theta}{2 \pi} ; A u t D=\left\{\lambda \phi_{a}\right\}$ is the group of 
automorphisms of $D$, with $\lambda \in \mathbb{T}, a \in D, \phi_{a}(z)=\frac{z-a}{1-\bar{a} z} ; \phi$ is inner if $\left|\phi^{*}\left(e^{i \theta}\right)\right|=1 \mathrm{~m}$-a.e., where $\phi^{*}\left(e^{i \theta}\right)$ is the radial limit of $\phi$ at $e^{i \theta}$; then, $\phi^{*}$ maps $\mathbb{T}$ to itself; finally, if $a \in D$, $P_{a}: \mathbb{T} \rightarrow \mathbb{R}^{+}$denotes the Poisson kernel at a: $P_{a}\left(e^{i t}\right)=\frac{1-|a|^{2}}{\left|e^{i t}-a\right|^{2}}$. The following facts are classical:

$$
H^{2} \text { is a Hilbert space of functions holomorphic in } D \text {. }
$$

$$
K_{a}(z)=\frac{1}{1-\bar{a} z} \text { is the reproducing kernel of } H^{2} \text { at } a ; \frac{K_{a}}{\left\|K_{a}\right\|} \stackrel{w}{\rightarrow} 0 \text { as }|a| \stackrel{<}{\rightarrow} 1 \text {. }
$$

$$
\text { For any } f \in H^{2},\|f\|^{2}=\sup _{0<r<1} \int\left|f\left(r e^{i \theta}\right)\right|^{2} d m=\int\left|f^{*}\left(e^{i \theta}\right)\right|^{2} d m .
$$

If $\phi(0)=0$ and if $u, v: D \rightarrow \mathbb{R}$ are subharmonic functions such that $v=u \circ \phi$, we say that $v$ is subordinate to $u$, and we have the famous [S2]:

THEOREM 1.1 (Littlewood's subordination principle). If $v$ is subordinate to $u$, then

$$
\int v\left(r e^{i \theta} d m \leq \int u\left(r e^{i \theta}\right) d m, \quad \text { for any } 0<r<1 .\right.
$$

Since $u=|f|^{2}$ is subharmonic when $f \in H^{2}$, (6) immediately gives

$$
\text { If } \phi(0)=0, C_{\phi} \text { is a contraction of } H^{2} \text { to itself. }
$$

Note that the converse is true: $(2)$ implies $C_{\phi}^{*}(1)=K_{\phi(0)}$, so that $\left(1-|\phi(0)|^{2}\right)^{-1 / 2}=$ $\left\|K_{\phi(0)}\right\| \leq 1$, and that $\phi(0)=0$.

The case $\phi(0)=a$ is handled by the following (easy to check) observations of Nordgren $[\mathrm{No}]$ :

$$
\text { If } \phi \text { is inner with } \phi(0)=a \text {, we have } \phi^{*}(m)=P_{a} m \text {. }
$$

In particular, if $f \in H^{2}$, we have that

$$
\int\left|f\left(\phi^{*}\left(e^{i \theta}\right)\right)\right|^{2} d m=\int \mid f\left(\left.e^{i \theta}\right|^{2} P_{a}\left(e^{i \theta}\right) d m,\right.
$$

and Nordgren used this to prove that

$$
\text { If } \phi \text { is inner with } \phi(0)=a \text {, we have }\left\|C_{\phi}\right\|=\left\|P_{a}\right\|_{\infty}=\left(\frac{1+|a|}{1-|a|}\right)^{1 / 2} \text {. }
$$

The two facts $(7),(10)$ combine to give:

$$
\text { For any } \phi, C_{\phi} \text { maps } H^{2} \text { to itself, and }\left\|C_{\phi}\right\| \leq\left(\frac{1+|\phi(0)|}{1-|\phi(0)|}\right)^{1 / 2} \text {. }
$$

Indeed, if $\phi(0)=a$, we have $\phi_{a} \circ \phi(0)=0$ and $\phi=\phi_{-a}\left(\phi_{a} \phi\right)$, so that $C_{\phi}=C_{\phi_{a} \circ \phi} C_{\phi_{-a}}$ and $\left\|C_{\phi}\right\| \leq\left\|C_{\phi_{-a}}\right\|=\left(\frac{1+|a|}{1-|a|}\right)^{1 / 2}$. The question of boundedness (not of the exact value of $\left\|C_{\phi}\right\|$ ! see [Co]) is thus automatically settled; and J. Shapiro [S1] added the following nice specification to (10) (if $T$ is an operator on $H^{2}$, we denote by $\|T\|_{e}$ its essential norm, i.e. its distance to compact operators):

THEOREM 1.2 (J. Shapiro). If $\phi$ is inner with $\phi(0)=a$, we have

$$
\left\|C_{\phi}\right\|_{e}=\left\|C_{\phi}\right\|=\left(\frac{1+|a|}{1-|a|}\right)^{1 / 2} .
$$


Proof. Let $K_{n}(n=0,1, \ldots)$ be the $n$-th partial sum operator: $K_{n} f(z)=\sum_{j=0}^{n} \hat{f}(j) z^{j}$, and let $R_{n}=I-K_{n}$ be the complementary orthogonal projection; $R_{n}$ is a norm-one, self-adjoint, operator, and $R_{n} f \rightarrow 0$ for each $f \in H^{2}$, so that it easily follows (see [S1] for the details) that, for any operator $T$, we have

$$
\|T\|_{e}=\lim _{n \rightarrow \infty}\left\|T R_{n}\right\| .
$$

Now, let $f \in H^{2}$, with $\|f\|_{2} \leq 1$; if $g(z)=z^{n+1} f(z)$, we have $R_{n} g=g$, so that (8) gives: $\left\|C_{\phi} R_{n}\right\|^{2} \geq\left\|C_{\phi} R_{n} g\right\|^{2}=\left\|C_{\phi} g\right\|^{2}=\int\left|g\left(e^{i \theta}\right)\right|^{2} P_{a}\left(e^{i \theta}\right) d m=\int\left|f\left(e^{i \theta}\right)\right|^{2} P_{a}\left(e^{i \theta}\right) d m=$ $\left\|C_{\phi} f\right\|^{2}$, showing that $\left\|C_{\phi} R_{n}\right\|^{2}=\left\|C_{\phi}\right\|^{2}$ for any $n$; now, (12) and (10) give the result.

The integral formula (5) was adapted, through the Littlewood subordination principle, to the study of boundedness for $C_{\phi}$; for the study of compactness, we need another integral representation, which is given by (see [S1], [S2]):

THEOREM 1.3 (Littlewood-Paley identity). For any $f \in H^{2}$, we have

$$
\|f\|^{2}=|f(0)|^{2}+\int_{D}\left|f^{\prime}(z)\right|^{2} d \lambda_{1}(z),
$$

where $d \lambda_{1}(z)$ is the probability measure $\frac{2}{\pi} \log \frac{1}{|z|} d x d y$ on $D$.

Using the change of variables formula in the non-injective case [Fe], Theorem 1.3 easily implies the following:

$$
\left\|C_{\phi} f\right\|^{2}=|f(\phi(0))|^{2}+\int_{D} \frac{N_{\phi}(w)}{\log \frac{1}{|w|}} d \lambda_{1}(w), \quad \text { for any } f \in H^{2},
$$

where $N_{\phi}$ is the so-called "Nevanlinna counting function":

$$
N_{\phi}(w)=\sum_{\phi(z)=w} \log \frac{1}{|z|} \quad\left(N_{\phi}(w)=0 \text { if } w \notin \phi(D)\right) .
$$

$N_{\phi}$ satisfies the Littlewood inequality:

$$
N_{\phi}(w) \leq \log \left|\frac{1-\bar{w} \phi(0)}{w-\phi(0)}\right|, \quad w \in D .
$$

(13) and (14) show part of the following

Theorem 1.4. Let $\phi \in H(D, D)$. Then:

a) If $C_{\phi}$ is compact, we have $\lim _{|z| \rightarrow 1} \frac{1-|\phi(z)|}{1-|z|}=\infty$.

b) The converse of a) is true if $\phi$ is injective, or finitely valent.

c) If $\|\phi\|_{\infty}<1, C_{\phi}$ is compact, and even in any Schatten class $S_{p}, p>0$.

d) $C_{\phi}$ is Hilbert-Schmidt $\left(\in S_{2}\right)$ if and only if $\int \frac{d \theta}{1-\left|\phi^{*}\left(e^{i \theta}\right)\right|} d m<\infty$.

Proof. a) We know from (4) that $K_{a} /\left\|K_{a}\right\| \stackrel{w}{\rightarrow} 0$, so $\left\|C_{\phi}^{*}\left(K_{a} /\left\|K_{a}\right\|\right)\right\|=\left\|K_{\phi(a)}\right\| /\left\|K_{a}\right\|$ $\rightarrow 0$ as $|a| \rightarrow 1$.

b) $\phi$ is finitely valent if there exists an integer $p$ such that, for any $w \in D$, the equation $\phi(z)=w$ has at most $p$ solutions (e.g. a Blaschke product of $p$ Möbius factors is exactly 
$p$-valent); if moreover $\frac{1-|\phi(z)|}{1-|z|} \rightarrow \infty$, we have

$$
\lim _{|w| \rightarrow 1} \frac{N_{\phi}(w)}{\log \frac{1}{|w|}}=0
$$

which is seen to be a sufficient condition (in fact also necessary) for the compactness of $C_{\phi}$ by using (13) (see [S1]). But (see [S2]) there are infinite Blaschke products $B$, for which $C_{B}$ is not compact since $B$ is inner, and such that $\lim _{|z| \rightarrow 1} \frac{1-|B(z)|}{1-|z|}=\infty$.

c) This is trivial, since the $n^{t h}$ approximation number $s_{n}$ of $C_{\phi}$ is $O\left(\|\phi\|_{\infty}^{n}\right)$, so that $\sum s_{n}^{p}<\infty$ for any $p>0$.

d) Equivalently, we have

$$
\int \frac{d m(\theta)}{1-\left|\phi^{*}\left(e^{i \theta}\right)\right|^{2}}=\sum_{0}^{\infty} \int\left|\phi^{*}\left(e^{i \theta}\right)\right|^{2 n} d m=\|\phi\|_{H S}^{2}<\infty .
$$

Let us mention that b) was used by MacCluer and Shapiro [McClS] to prove the existence of a surjective (and finitely valent) $\phi: D \rightarrow D$ such that $C_{\phi}$ is yet compact.

Theorem 1.4 has the important

Corollary 1.5. a) There are compact composition operators $C_{\phi}$ for which $\|\phi\|_{\infty}=1$.

b) There exist compact, but non-Hilbert-Schmidt, composition operators $C_{\phi}$ (then $\left.\|\phi\|_{\infty}=1\right)$.

Proof. a) Let $P$ be a polygon inscribed in $D$, and let $\phi: D \rightarrow P$ be a conformal mapping. We have $\|\phi\|_{\infty}=1$, and it is fairly easy (see [S2], or use the Schwarz-Christoffel formulae) to check that $\int \frac{d m}{1-\mid \phi^{*}\left(e^{i \theta} \mid\right.}<\infty$, so that $C_{\phi}$ is even Hilbert-Schmidt.

b) This is more delicate, and we refer to [CoMcCl] pp. 147-148 for the details.

The general necessary and sufficient condition (already mentioned in b) of Theorem 1.4) was found by Shapiro [S1], in a beautiful way:

THEOREM 1.6. A necessary and sufficient condition for $C_{\phi}: H^{2} \rightarrow H^{2}$ to be compact is that $N_{\phi}(w)=o\left(\log \frac{1}{|w|}\right)$ as $|w| \stackrel{\leq}{\longrightarrow}$. More precisely, we have

$$
\left\|C_{\phi}\right\|_{e}=\varlimsup_{|w| \rightarrow 1}\left(\frac{N_{\phi(w)}}{\log \frac{1}{|w|}}\right)^{1 / 2} .
$$

The characterization of compactness for composition operators on $H^{2}$ is thus seen to be a significant result, although it is completely solved. The situation is not so satisfactory with Dirichlet series, as we shall see in Section 4. Let us finish with the somewhat simpler problems of invertibility or isometry on $H^{2}$.

ThEOREM 1.7 ([McCl]). For $\phi \in H(D, D)$, the following are equivalent:

a) $C_{\phi}$ is Fredholm;

b) $C_{\phi}$ is invertible;

c) $\phi \in$ Aut D.

Proof. a) $\Rightarrow \mathrm{c}$ ). If $\phi(a)=\phi(b)$ for some distinct $a, b \in D$, the open mapping theorem shows the existence of disjoint sequences $\left(a_{n}\right),\left(b_{n}\right)$ of distinct points such that $\phi\left(a_{n}\right)=$ 
$\phi\left(b_{n}\right)$, with $a_{n} \rightarrow a, b_{n} \rightarrow b$. Then, the functions $K_{a_{n}}-K_{b_{n}}$ are linearly independent and in the kernel $N$ of $C_{\phi}^{*}$, so that $\operatorname{dim} N=\infty$, contradicting the Fredholm character of $C_{\phi}^{*}$, and showing that $\phi$ is injective. If $\phi$ is not surjective, a connectedness argument shows that the boundary of $\phi(D)$ intersects $D$, and we can find $v \in D$ and a sequence $\left(z_{n}\right)$ such that $\left|z_{n}\right| \rightarrow 1$ and $\phi\left(z_{n}\right) \rightarrow v$. Set $T=C_{\phi}^{*}, f_{n}=K_{z_{n}} /\left\|K_{z_{n}}\right\|$.

$T f_{n}=K_{\phi\left(z_{n}\right)} /\left\|K_{z_{n}}\right\| \stackrel{s}{\rightarrow} 0$, and $f_{n} \stackrel{w}{\rightarrow} 0$. If $L, S$ are a compact and a bounded operator, we have $S T f_{n} \stackrel{s}{\rightarrow} 0$, whereas $\left\|(I+L) f_{n}\right\| \rightarrow 1$, preventing the relation $S T=I+L$, i.e. preventing $T$ to be invertible modulo compact operators, i.e. preventing [Ar] $T$ to be Fredholm. So $\phi$ is surjective, and we are done, since c) $\Rightarrow$ b) and b) $\Rightarrow$ a) are trivial.

As concerns isometries, we have

THEOREM 1.8. For $\phi \in H(D, D)$, the following are equivalent:

a) $C_{\phi}: H^{2} \rightarrow H^{2}$ is (similar to) an isometry.

b) $\phi$ is inner and fixes the origin.

Proof. b) $\Rightarrow$ a) is an immediate consequence of (8). Conversely, if $C_{\phi}$ is an isometry, we have, using (8) and (7), $\phi(0)=0$, and so $1=\left\|C_{\phi}(z)\right\|^{2}=\int\left|\phi^{*}\left(e^{i \theta}\right)\right|^{2} P_{0}\left(e^{i \theta}\right) d m=$ $\int\left|\phi^{*}\left(e^{i \theta}\right)\right|^{2} d m$, implying that $\phi$ is inner. If we only assume that $C_{\phi}$ is similar to an isometry, things are slightly more complicated, and we refer to $[\mathrm{J}]$ or $[\mathrm{B} 1]$.

2. The Wiener algebra $A^{+}$. We recall that $A^{+}$is the Banach algebra of functions $f(z)=\sum_{0}^{\infty} a_{n} z^{n}$ which are analytic in $D$ and have summable coefficients: $\|f\|=$ $\sum_{0}^{\infty}\left|a_{n}\right|<\infty$.

$A^{+}$is a commutative, unital, Banach algebra with spectrum $\bar{D}$. In contrast to the case of $H^{2}$, if $\phi \in H(D, D)$, it is not automatic that $C_{\phi}$ is bounded on $A^{+}$, and we have the following nice necessary and sufficient condition due to Newman [Ne].

THEOREM 2.1. The following are equivalent:

a) $C_{\phi}$ maps $A^{+}$to itself;

b) $\phi \in A^{+}$and $\left\|\phi^{n}\right\|_{A^{+}}=O(1)$ as $n \rightarrow \infty$; this happens if and only if all maximum points $\theta_{0}$ of $\left|\phi\left(e^{i \theta}\right)\right|$ are "ordinary points", i.e. if and only if we have, as $t \rightarrow 0$,

$$
\log \phi\left(e^{i\left(\theta_{0}+t\right)}\right)=\alpha_{0}+\alpha_{1} t+\alpha_{k} t^{k}+\ldots,
$$

where $k>1$ and $\alpha_{k} \neq 0$ is not purely imaginary.

Newman used this theorem to give the following two non-trivial examples:

ExAmple 1. $\phi(z)=\frac{1+z-z^{2}}{\sqrt{5}} \Rightarrow C_{\phi}$ maps $A^{+}$to $A^{+}$, and $\|\phi\|_{\infty}=1$.

Indeed, writing $z \in \mathbb{T}$ under the form $z=c+i s, c=\cos t, s=\sin t$, we have $\left|1+z-z^{2}\right|=|\bar{z}+1-z|=|1-2 i s|=\sqrt{1+4 s^{2}} \leq \sqrt{5}$. The maximum points are $\theta_{0}= \pm \frac{\pi}{2}$, and they "pass" the criterion (15), see [Ne], p. 39.

EXAMPLE 2. $\phi(z)=\frac{12+16 z-3 z^{2}}{25} \Rightarrow C_{\phi}$ does not map $A^{+}$to itself.

Indeed, $\left\|\phi_{\infty}\right\|=1$ and $\theta_{0}=0$ is the only maximum point, due to the identity

$$
\left|12+16 z-3 z^{2}\right|^{2}+36|z-1|^{4}=625,
$$


for any $z$ of modulus one. In fact, if we write $z=c+i s, c=\cos t, s=\sin t$ we have

$$
\begin{aligned}
\mid 12+16 z- & \left.3 z^{2}\right|^{2}+36|z-1|^{4}=|12 \bar{z}+16-3 z|^{2}+36|z-1|^{4}=|9 c+16-15 i s|^{2} \\
+ & 144(c-1)^{2}=(9 c+16)^{2}+225 s^{2}+144(c-1)^{2}=(81+144) c^{2} \\
+ & 288 c-288 c+225 s^{2}+256+144=225+256+144=625 .
\end{aligned}
$$

And $\theta_{0}=0$ fails to pass (see $[\mathrm{Ne}]$ p. 40) the criterion (2.1).

Compactness was not studied by Newman, but we can easily [BFLQ1] prove the following necessary and sufficient condition, similar to the result of [Sc] and [B2] for the algebras $H^{\infty}$ and $\mathcal{H}^{\infty}$.

THEOREM 2.2 ([BFLQ1]). For a non-constant analytic function $\phi: D \rightarrow \bar{D}$ inducing a bounded $C_{\phi}: A^{+} \rightarrow A^{+}$, the following are equivalent: a) $C_{\phi}$ is compact; b) $\|\phi\|_{\infty}<1$.

Proof. It is easily seen that $C_{\phi}$ is compact if and only if $\left\|\phi^{n}\right\|_{A^{+}} \rightarrow 0$; and we have, by the spectral radius formula

$$
\|\phi\|_{\infty}=\lim _{n \rightarrow \infty}\left\|\phi^{n}\right\|_{A^{+}}^{1 / n}=\inf _{n \geq 1} \|\left.\phi^{n}\right|_{A^{+}} ^{1 / n},
$$

giving the result.

Newman proved that the composition operators on $A^{+}$are very poor in automorphisms (i.e. in invertible composition operators) since we have

Theorem 2.3. For a bounded $C_{\phi}: A^{+} \rightarrow A^{+}$, the following are equivalent:

a) $C_{\phi}$ is invertible;

b) $\phi$ is a rotation: $\phi(z)=\lambda z$, with $|\lambda|=1$.

Proof. a) $\Rightarrow$ b. As in the case of $H^{2}$, it is easy to see that we must have $\phi \in A u t D$, i.e. $\phi(z)=\lambda \frac{z-a}{1-\bar{a} z}$, with $\lambda \in \mathbb{T}$ and $a \in D$.

But here, a big difference with $H^{2}$ occurs:

LEMmA 2.4. Let $\phi \in A^{+}$, with $\phi\left(e^{i t}\right)=e^{i g(t)}, g$ being a real $C^{2}$, non-affine function. Then

$$
\left\|\phi^{n}\right\|_{A^{+}} \geq \delta \sqrt{n}, \quad \text { for some positive constant } \delta .
$$

This is proved in [Kal] p. 76, as a consequence of the van der Corput inequalities for integrals, and is sharp: we also have $\left\|\phi^{n}\right\|_{A^{+}} \leq C \sqrt{n}$.

Now, if $\phi(z)=\lambda \frac{z-a}{1-\bar{a} z}$ and if $\mathbb{C}_{\phi}$ maps $A^{+}$to itself, Theorem 2.1 shows that $\left\|\phi^{n}\right\|_{A^{+}}=$ $O(1)$, and since $\phi\left(e^{i t}\right)$ is unimodular, Lemma 2.4 shows that $g$ has to be affine, so that $a=0$, which finishes the proof of Theorem 2.3.

The composition operators on $A^{+}$are also poor in isometries, and Harzallah (see the book of [Kal] p. 144) obtained the following

TheOREM 2.5. For a bounded $C_{\phi}: A^{+} \rightarrow A^{+}$, the following are equivalent:

a) $C_{\phi}$ is isometric;

b) $\phi$ is a monomial $\phi(z)=\lambda z^{d}$, with $|\lambda|=1$, and $d \in \mathbb{N}$. 
We have only to prove that a) $\Rightarrow$ b); this is now a special case of a more general result, which we shall prove in Section 5. We see in that section that, unlike the case of $H^{2}$, the main problem here is not compactness, but boundedness.

3. General facts about Dirichlet series. Recall that a Dirichlet series is a series of the form

$$
A(s)=\sum_{1}^{\infty} a_{n} n^{-s}, \text { with } s=\sigma+i t \in \mathbb{C} .
$$

The analogue of the radius of convergence for Taylor series is here the abscissa of convergence, but one should bear in mind the fact that there are several such abscissas (see e.g. [Q2]): the abscissa $\sigma_{c}$ of simple convergence: (18) converges for $\Re s>\sigma_{c}$, diverges for $\Re s<\sigma_{c}$; the abscissa $\sigma_{u}$ of uniform convergence: (18) converges uniformly in $\Re s \geq \sigma_{u}+\varepsilon$, not in $\Re s \geq \sigma_{u}-\varepsilon$; the abscissa $\sigma_{a}$ of absolute convergence: (18) converges absolutely in $\Re s \geq \sigma_{a}+\varepsilon$, not in $\Re s \geq \sigma_{a}-\varepsilon$; the abscissa $\sigma_{h}$ of holomorphy: $A$ has an analytic extension to $\Re s>\sigma_{h}$, not to $\Re s>\sigma_{h}-\varepsilon$.

Those abscissas are related by $\sigma_{h} \leq \sigma_{c} \leq \sigma_{u} \leq \sigma_{a}$, and we have (see [Q2])

$$
\begin{aligned}
\sigma_{a} & \leq \sigma_{u}+\frac{1}{2} \quad \text { (optimal); } \\
\sigma_{a} \leq \sigma_{c}+1 & \text { (optimal). }
\end{aligned}
$$

The difference $\sigma_{a}-\sigma_{c}$ can take any value between 0 and 1: indeed, if $0<\alpha<1$ and if $a_{n}=e^{i n^{\alpha}}$, the Euler-Maclaurin summation formula shows that $\sum_{k=1}^{n} e^{i k^{\alpha}} \sim \frac{n^{1-\alpha}}{i \alpha} e^{i n^{\alpha}}$, so that $\sigma_{a}=1$ and $\sigma_{c}=1-\alpha$.

The inequality $\sigma_{h} \leq \sigma_{c}$ may be strict, whereas a Taylor series always has a singular point on its circle of convergence; more precisely:

Proposition 3.1. There exists a Dirichlet series $\sum_{1}^{\infty} a_{n} n^{-s}=A(s)$ such that $\sigma_{c}=0$ and $\sigma_{h}=-\infty$.

Proof. One possibility is to take the alternate Riemann series $\sum_{1}^{\infty}(-1)^{n-1} / n^{s}=$ $\left(1-2^{1-s}\right) \zeta(s)$; the zero of the first factor kills the unique pole of zeta at 1 , so that $\sigma_{h}=-\infty$, and clearly $\sigma_{c}=0$; any L-function associated with a non-principal character modulo $q \geq 3$ has the same property; but perhaps the following example, shown to me by $\mathrm{J}$. Peyrière $[\mathrm{P}]$ some years ago, is the most elementary: let $\left(\varepsilon_{n}\right)_{n \geq 0}$ be the Morse sequence, defined by $\varepsilon_{0}=1, \varepsilon_{2 n}=\varepsilon_{n}, \varepsilon_{2 n+1}=-\varepsilon_{n}$, for which

$$
\sum_{0}^{\infty} \varepsilon_{n} z^{n}=(1-z)\left(1-z^{2}\right)\left(1-z^{4}\right) \ldots \quad \text { for } z \in D .
$$

Consider the Dirichlet series $A(s)=\sum_{0}^{\infty} \varepsilon_{n} /(n+1)^{s}$. Since

$$
\frac{1}{(n+1)^{s}}=\frac{1}{\Gamma(s)} \int_{0}^{\infty} t^{s-1} e^{-n t} e^{-t} d t, \quad \Re s>0,
$$

we clearly have

$$
A(s)=\frac{1}{\Gamma(s)} \int_{0}^{\infty} t^{s-1} \prod_{k=0}^{\infty}\left(1-e^{-2^{k} t}\right) e^{-t} d t, \quad \text { for } \Re s>1 .
$$


The RHS of (19) is an entire function, since the product in the integrand has a zero of infinite order at $t=0$; therefore, $\sigma_{h}=-\infty$. If we set $S_{n}=\varepsilon_{0}+\cdots+\varepsilon_{n}$, we have $S_{2 n+1}=\sum_{k=0}^{n}\left(\varepsilon_{2 k}+\varepsilon_{2 k+1}\right)=\sum_{k=0}^{n}\left(\varepsilon_{k}-\varepsilon_{k}\right)=0$, so that $\left|S_{n}\right| \leq 1$ for each $n$, and an Abel summation by parts shows that $\sigma_{c}=0$.

In Section 4, we shall encounter $\ell_{2}$-series $A(s)=\sum_{1}^{\infty} a_{n} n^{-s}$ with square-summable coefficients; for such series, $\sigma_{a} \leq \frac{1}{2}$, since we have by Cauchy-Schwarz: $\sum_{1}^{\infty}\left|a_{n} n^{-s}\right|$ $\leq\left(\sum_{1}^{\infty}\left|a_{n}\right|^{2}\right)^{1 / 2}\left(\sum_{1}^{\infty} n^{-2 \sigma}\right)^{1 / 2}$. The existence of "exotic" such series will be useful to us, under the form of the following theorem, in which $\left(\varepsilon_{n}\right)$ denotes a sequence of independent, symmetric, equidistributed, non-zero, square-integrable random variables defined on some probability space $\Omega$.

THEOREM 3.2. a) Let $\left(b_{n}\right)_{n \geq 1}=n^{-1 / 2}(\log (n+1))^{-1}$ and $A_{\omega}(s)=\sum_{1}^{\infty} \varepsilon_{n}(\omega) b_{n} n^{-s}$. Then, we have almost surely that $\sigma_{c}\left(A_{\omega}\right)=0$ and that the line $\Re s=0$ is a natural boundary for the $l_{2}$-series $A_{\omega}$.

b) There exists a $\ell_{2}$-series $A$ such that $\sigma_{c}=\frac{1}{2}$ and that the line $\Re s=\frac{1}{2}$ is a natural boundary for $A$.

Proof. a) Since $\sum_{1}^{\infty} b_{n}^{2} n^{-2 \sigma}<\infty$ if and only if $\sigma \geq 0$, the three series theorem [Re] shows that $\sigma_{c}\left(A_{\omega}\right)=0$ almost surely; and since the variables $X_{n}=\varepsilon_{n} b_{n}$ are independent and symmetric, a general result [Ka2] or [LiQ] shows that the vertical line $\Re s=0$ is almost surely a natural boundary for $A_{\omega}$.

b) Instead of using a probabilistic method as in a), we shall now use a topological method, i.e. we shall apply the Baire category theorem to the compact space $\Omega=\{-1,1\}^{\mathbb{N}}$ of all choices of signs $\omega=\left(\varepsilon_{n}(\omega)\right)_{n \geq 1}, \varepsilon_{n}(\omega)= \pm 1$, equipped with its natural topology.

A subset $E$ of $\Omega$ will be said to be quasi-sure if $E$ contains a dense $G_{\delta}$ set of $\Omega$. The result will be a special case of the following theorem, where $0 \leq \lambda_{1}<\lambda_{2}<\ldots<\lambda_{n}<\ldots$, $\lambda_{n} \rightarrow \infty$.

THEOREM 3.3. Let the general Dirichlet series $\sum_{1}^{\infty} a_{n} e^{-\lambda_{n} s}$ have the abscissa of absolute convergence $\sigma_{a}=\alpha$; then, the line $\Re s=\alpha$ is quasi-surely a natural boundary for the series $f_{\omega}(s)=\sum_{1}^{\infty} \varepsilon_{n}(\omega) a_{n} e^{-\lambda_{n} s}, \omega \in \Omega$.

Proof (see [Q1], which contains a slight mistake; replace $\sigma_{c}$ by $\sigma_{a}$ ). Denote by $Q$ the set of rational numbers, by $E$ the set of $\omega \in \Omega$ for which $\Re s=\alpha$ is not a natural boundary for $f_{\omega}$. We have

$$
E=\bigcup E_{a, r, N}, \text { where } a=\alpha+i t, t \in Q, r \in Q^{+}, N \text { integer } \geq 1,
$$

and where $E_{a, r, N}$ is the set of $\omega$ 's such that $\sum_{1}^{\infty} \varepsilon_{n}(\omega) a_{n} e^{-\lambda_{n} s}$ has an analytic extension (still denoted by $\left.f_{\omega}\right)$ to $D(a, r)=\{s:|s-a|<r\}$, with $\left|f_{\omega}\right| \leq N$ for $s \in D(a, r)$.

A simple normal family argument shows that each $E_{a, r, N}$ is closed in $\Omega$; let $\omega_{0}=\left(\varepsilon_{n}\right)$ be an interior point of $E_{a, r, N}$, and $M$ an integer such that $\varepsilon_{n}(\omega)=\varepsilon_{n}$ for each $n \leq M$ implies $\omega \in E_{a, r, N}$; if now $\omega \in \Omega$, write $f_{\omega}(s)=\left[\sum_{1}^{M} \varepsilon_{n} a_{n} e^{-\lambda_{n} s}+\sum_{M+1}^{\infty} \varepsilon_{n}(\omega) a_{n} e^{-\lambda_{n} s}\right]+$ $\left[\sum_{1}^{M}\left(\varepsilon_{n}(\omega)-\varepsilon_{n}\right) a_{n} e^{-\lambda_{n} s}\right]=\left[f_{\omega^{\prime}}(s)\right]+[g(s)]$, where $\omega^{\prime} \in E_{a, r, N}$ and where the Dirichlet polynomial $g$ is an entire function. So that $f_{\omega}$ can be analytically extended to $D(a, r)$, whith $\left|f_{\omega}(s)\right| \leq N+\sum_{1}^{M}\left|a_{n}\right| e^{-\lambda_{n}(\alpha-r)} \stackrel{\text { def }}{=} C$. 
Now, take $0<\rho<\frac{r}{3}$, so that $\bar{D}(a+\rho, 2 \rho) \subset D(a, r)$. The Cauchy inequalities imply

$$
\frac{\left|f_{\omega}^{(j)}(a+\rho)\right|}{j !}=\frac{\left|\sum_{1}^{\infty} a_{n} \varepsilon_{n}(\omega) \lambda_{n}^{j} e^{-\lambda_{n}(a+\rho)}\right|}{j !} \leq \frac{C}{(2 \rho)^{j}}, \quad j=0,1, \ldots
$$

Since $\sup _{\varepsilon_{n}= \pm 1}\left|\sum_{1}^{\infty} \varepsilon_{n} z_{n}\right| \geq \frac{1}{2} \sum_{1}^{\infty}\left|z_{n}\right|$ for any sequence $\left(z_{n}\right)$ of complex numbers such that $\sum_{1}^{\infty}\left|z_{n}\right|<\infty$, we get

$$
\sum_{n=1}^{\infty} \frac{\left|a_{n}\right|^{j} \lambda_{n}^{j} e^{-\lambda_{n}(\alpha+\rho)}}{j !} \leq \frac{2 C}{(2 \rho)^{j}} .
$$

Take $\rho<R<2 \rho$, multiply each term of $(*)$ by $R^{j}, j=0,1, \ldots$, sum and permute to get

$$
\sum_{n=1}^{\infty}\left|a_{n}\right| e^{-\lambda_{n}(\alpha+\rho)} e^{\lambda_{n} R} \leq 2 C \sum_{j=0}^{\infty}\left(\frac{R}{2 \rho}\right)^{j}<\infty .
$$

Or $\sum_{n=1}^{\infty}\left|a_{n}\right| e^{-\lambda_{n}(\alpha+\rho-R)}<\infty$. But this is impossible since $\alpha+\rho-R<\alpha$. This contradiction shows that $E_{a, r, N}$ is of empty interior; therefore, $E$ is a dense $G_{\delta}$ set, and this ends the proof of Theorem 3.3. To derive b) of Theorem 3.2, start from $\sum_{1}^{\infty} \frac{1}{\sqrt{n} \log (n+1)} n^{-s}$; the choice $a_{n}=\frac{\varepsilon_{n}(\omega}{\sqrt{n} \log (n+1)}$ will work for some $\omega \in \Omega$ (chosen "topologically", not "at random" !)

4. The Hardy-Dirichlet space $\mathcal{H}^{2}$. This is the Hilbert space of Dirichlet series $f(s)=$ $\sum_{1}^{\infty} a_{n} n^{-s}$, with $\|f\|^{2}=\sum_{1}^{\infty}\left|a_{n}\right|^{2}<\infty$; it was introduced by Hedenmalm, Lindqvist, Seip [HLS] to study completeness problems in $L^{2}(0,1)$; an orthonormal basis of $\mathcal{H}^{2}$ is formed by the $e_{n}(s)=n^{-s}, n=1,2, \ldots$, so that the reproducing kernel $K_{a}$ of $\mathcal{H}^{2}$ at $a \in \mathbb{C}_{1 / 2}$ is: $K_{a}(s)=\sum_{1}^{\infty} e_{n}(s) \overline{e_{n}(a)}=\zeta(s+\bar{a})$, where $\zeta$ denotes the Riemann zeta function; this is enough to demonstrate that the functional properties of $\mathcal{H}^{2}$ will be fairly different from those of the Hardy space $H^{2}$ !

It will be convenient to introduce the space $\mathcal{D}$ of functions which are analytic in $\mathbb{C}_{1 / 2}$, and representable by a convergent Dirichlet series $\sum_{1}^{\infty} a_{n} n^{-s}$ for $\Re s$ large enough (a typical example is $f(s)=\Psi(s-a)$, where $\Psi(s)=\left(1-2^{1-s}\right) \zeta(s) ; f$ is entire, and representable by $\sum_{1}^{\infty}(-1)^{n-1} n^{a} n^{-s}$ for $\left.\Re s>a\right)$.

If $f \in \mathcal{D}, f(s)=\sum_{1}^{\infty} a_{n} n^{-s}$, we have $f(s) \rightarrow a_{1}$ as $\Re s \rightarrow \infty$, so that there are analytic functions $\left(s, e^{s}, \ldots\right)$ analytic on $\mathbb{C}_{1 / 2}$ which do not belong to $\mathcal{D}$.

This leads to a definition: An analytic function $\phi$ on $\mathbb{C}_{1 / 2}$ will be called representable if $\phi(s)=c_{0} s+\varphi(s)$, where $c_{0}$ is a non-negative integer and $\varphi \in \mathcal{D}$.

The following two results were proved in [GH]: Recall that $\mathbb{C}_{\theta}$ denotes the half-plane $\Re s>\theta$, and that $\mathbb{N}=\{1,2, \ldots\}$ and $N_{0}=\mathbb{N} \cup\{0\}$.

THEOREM 4.1. Let $\phi: \mathbb{C}_{0} \rightarrow \mathbb{C}$ be an analytic function such that $k^{-\phi} \in \mathcal{D}$ for $k=$ $1,2, \ldots$ Then, $\phi$ is representable.

THEOREM 4.2. An analytic self-map $\phi: \mathbb{C}_{1 / 2} \rightarrow \mathbb{C}_{1 / 2}$ induces a bounded composition operator $C_{\phi}: f \mapsto f \circ \phi$ on $\mathcal{H}^{2}$ if and only if 
a) $\phi$ is representable: $\phi(s)=c_{0} s+\varphi(s)$, with $c_{0} \in \mathbb{N}_{0}$ and $\varphi \in \mathcal{D}$.

b) $\phi$ is "extendable" with "controlled range", namely $\phi$ has an analytic extension to $\mathbb{C}_{0}$, still denoted by $\phi$, and such that

i) $\phi\left(\mathbb{C}_{0}\right) \subset \mathbb{C}_{0}$ if $c_{0} \geq 1$.

ii) $\phi\left(\mathbb{C}_{0}\right) \subset \mathbb{C}_{1 / 2}$ if $c_{0}=0$.

It will be convenient to introduce also the set $S$ of all completely multiplicative functions $\chi: \mathbb{N} \rightarrow \mathbb{T}$ such that $\chi(1)=1$; such a function is completely determined by the sequence $\left(\chi\left(p_{1}\right), \chi\left(p_{2}\right), \ldots\right) \in \mathbb{T}^{\infty}$ of its values on prime numbers $p_{1}, p_{2}, \ldots$, so $S$ may and will be identified with $\mathbb{T}^{\infty}$, equipped with its Haar measure $m$.

If $f(s)=\sum_{1}^{\infty} a_{n} n^{-s} \in \mathcal{H}^{2}$ and $\chi \in S$, we write

$$
f_{\chi}(s)=\sum_{1}^{\infty} a_{n} \chi(n) n^{-s} .
$$

The following result is due to Helson [He]. Using a theorem of Menchoff [Al], one can give a simplified proof [B3].

THEOREM 4.3. Let $f(s)=\sum_{1}^{\infty} a_{n} n^{-s} \in \mathcal{H}^{2}$. Then, for almost all $\chi \in S, f_{\chi}$ has an analytic extension to $\mathbb{C}_{0}$.

Proof. Set $f_{n}(\chi)=\chi(n)$. The functions $f_{n}: S \rightarrow \mathbb{C}$ are not independent random variables, but they form an orthonormal system. For such a system, the theorem of Menchoff reads:

$(*)$ If $c_{1}, \ldots, c_{n} \ldots$ are complex numbers such that $\sum_{1}^{\infty}\left|c_{n}\right|^{2} \log ^{2} n<\infty$, then $\sum_{1}^{\infty} c_{n} f_{n}(\chi)$ converges for almost all $\chi$.

Applying $(*)$ with $c_{n}=a_{n} n^{-s}, s \in \mathbb{C}_{0}$, and letting $s$ take the values $\frac{1}{2}, \frac{1}{3}, \ldots$, we immediately get Theorem 4.3.

In Section 2, the integral representations for the norm (e.g. the Littlewood-Paley identity) played an important role; such representations are more difficult to obtain here, but we still have (see [GH] and [B2]):

Proposition 4.4. a) Let $u \in L^{1}(\mathbb{R})$, with $u \geq 0$ and $\|u\|_{1}=1$. Set $u_{a}(t)=a^{-1} u\left(t a^{-1}\right)$, $a>0$. If $f(s)=\sum_{1}^{\infty} a_{n} n^{-s} \in \mathcal{H}^{2}$, the series being uniformly convergent in $\mathbb{C}_{0}$, we have

$$
\|f\|^{2}=\sum_{1}^{\infty}\left|a_{n}\right|^{2}=\lim _{a \rightarrow+\infty} \int_{-\infty}^{\infty}|f(i t)|^{2} u_{a}(t) d t .
$$

b) For any $f \in \mathcal{H}^{2}$ and any Borel probability measure $\mu$ on $\mathbb{R}$, we have

$$
\|f\|^{2}=|f(\infty)|^{2}+\int_{0}^{\infty} \int_{\mathbb{R}} \int_{\mathbb{T}} \sigma\left|f_{\chi}^{\prime}(\sigma+i t)\right|^{2} d \sigma d \mu(t) d m(\chi) .
$$

We can now sketch the proof of Theorem 4.2.

We first show that the conditions on $\phi$ are sufficient. If $c_{0} \geq 1$, for $a>0$, denote by $\Psi_{a}$ the conformal mapping $s \mapsto \frac{s-a}{s+a}$ of $\mathbb{C}_{0}$ onto $D$, and by $\lambda_{a}$ the probability measure $\frac{a}{\pi\left(a^{2}+t^{2}\right)} d t$ on $\mathbb{R}$, for which one easily checks that $\lambda_{a}=\Psi_{a}^{-1}(m), m$ being the Haar measure of $\mathbb{T}$. 
Now, let $f(s)=\sum_{1}^{N} a_{n} n^{-s}$ be a Dirichlet polynomial, and $a, b>0$; we have from b) that $\omega=\Psi_{b} \circ \phi \circ \Psi_{a}^{-1} \in H(D, D)$ and $F=f \circ \Psi_{b}^{-1} \in H^{2}$, the Hardy space ( $F$ is bounded); by (1.9) of Section 1, we have

$$
\int|F \circ \omega|^{2} d m \leq \frac{1+|\omega(0)|}{1-|\omega(0)|} \int|F|^{2} d m,
$$

equivalently

$$
\int|f \circ \phi|^{2} d \lambda_{a} \leq \frac{1+\mid \frac{\phi(a)-b}{\phi(a)+b \mid}}{1-\mid \frac{\phi(a)-b}{\phi(a)+b \mid}} \int|f|^{2} d \lambda_{b} .
$$

Observe (this is due to a)) that $\phi(a) \sim c_{0} a$ as $a \rightarrow+\infty$, then take $b=c_{0} a$, let $a \rightarrow \infty$ and apply a) of Proposition 4.4 to $u(t)=\frac{1}{\pi\left(1+t^{2}\right)}$ to get (\| $\|$ denoting the norm in $\mathcal{H}^{2}$ ) from (24): $\|f \circ \phi\|^{2} \leq\|f\|^{2}$.

This shows that $C_{\phi}: \mathcal{H}^{2} \rightarrow \mathcal{H}^{2}$ is even a contraction.

If $c_{0}=0$, things are slightly more complicated. We use the inequality

$$
\int_{\alpha}^{\alpha+1}\left|\sum_{1}^{N} a_{n} n^{i t}\right|^{2} d t \leq C \sum_{1}^{N} n\left|a_{n}\right|^{2}
$$

where $\alpha \in \mathbb{R}$ and $C$ is a numerical constant, which is an easy consequence of the weighted Hilbert inequality [MVau], [MVaa], in which $\lambda_{1}, \ldots, \lambda_{N}$ denote distinct real numbers and $\delta_{n}=\inf _{m \neq n}\left|\lambda_{m}-\lambda_{n}\right|:$

$$
\left|\sum_{m \neq n} \frac{a_{m} \overline{a_{n}}}{\lambda_{m}-\lambda_{n}}\right| \leq C_{0} \sum \frac{\left|a_{n}\right|^{2}}{\delta_{n}} .
$$

One easily derives from (25) that

$$
\int|f(\sigma+i t)|^{2} d \lambda_{a}(t) \leq C\|f\|^{2}, \quad \forall f \in \mathcal{H}^{2}, \forall a>0, \forall \sigma>\frac{1}{2} .
$$

In fact, with obvious notations,

$$
\begin{gathered}
\int|f(\sigma+i t)|^{2} d \lambda_{a}(t) \ll \sum_{k \in \mathbb{Z}} \int_{k}^{k+1}|f(\sigma+i t)|^{2} \frac{a}{a^{2}+t^{2}} d t \\
\ll \sum_{k \in \mathbb{Z}} \frac{a}{a^{2}+k^{2}} \quad \int_{k}^{k+1}|f(\sigma+i t)|^{2} d t \ll \sum_{k \in \mathbb{Z}} \frac{a}{a^{2}+k^{2}} \sum_{n=1}^{\infty} n n^{-2 \sigma}\left|a_{n}\right|^{2} \\
\quad\left(\text { by }(25) \text { applied to } f(\sigma+i t)=\sum a_{n} n^{-\sigma} n^{-i t}\right) \\
\ll \sum_{k \in \mathbb{Z}} \frac{a}{a^{2}+k^{2}}\|f\|^{2} \ll\|f\|^{2} \int \frac{a d t}{a^{2}+t^{2}} \ll\|f\|^{2} .
\end{gathered}
$$

We now argue as in the case $c_{0} \geq 1$, but we take $\omega=\Psi_{b} \circ \tau \circ \phi_{\epsilon} \circ \Psi_{a}^{-1}, \quad F=f \circ \tau^{-1} \circ \Psi_{b}^{-1}$, where $\phi_{\epsilon}(s)=\phi(s+\epsilon), \epsilon>0$, and where $\tau(s)=s-1 / 2$. We get, using the inequality

$$
\int|F \circ \omega|^{2} d m \leq \frac{1+|\omega(0)|}{1-|\omega(0)|} \int|F|^{2} d m
$$

the facts that $F \circ \omega=f \circ \phi_{\epsilon} \circ \Psi_{a}^{-1}, \lambda_{a}=\Psi_{a}^{-1}(m), \lambda_{b}=\Psi_{b}^{-1}(m)$, and finally (26) (where 
we let $\sigma$ tend to $\frac{1}{2}$ when $f$ is a Dirichlet polynomial), that

$$
\int\left|f \circ \phi_{\varepsilon}(i t)\right|^{2} d \lambda_{a}(t) \leq \frac{1+|\omega(0)|}{1-|\omega(0)|} \int\left|f\left(\frac{1}{2}+i t\right)\right|^{2} d \lambda_{b}(t) \leq C \frac{1+|\omega(0)|}{1-|\omega(0)|}\|f\|^{2} .
$$

Write $f \circ \phi(s)=\sum_{n=1}^{\infty} b_{n} n^{-s} ; f \circ \phi$ is bounded in $\mathbb{C}_{0}$, so by a famous theorem of Bohr [Bo], the series $\sum_{1}^{\infty} b_{n} n^{-s}$ converges uniformly in each half-plane $\mathbb{C}_{\varepsilon}$; so that if we let $a \rightarrow \infty$ (keeping $\varepsilon$ and $b$ fixed) above, we get from $(22)$, since $\omega(0)=\Psi_{b}\left[\phi(a+\varepsilon)-\frac{1}{2}\right] \rightarrow$ $\Psi_{b}\left(c_{1}-\frac{1}{2}\right)=z_{b} \in D$,

$$
\sum_{1}^{\infty}\left|b_{n}\right|^{2} n^{-2 \varepsilon} \leq C \frac{1+\left|z_{b}\right|}{1-\left|z_{b}\right|}\|f\|^{2}=C_{b}\|f\|^{2} .
$$

Now, let $\varepsilon$ tend to zero to get $\|f \circ \phi\|^{2}=\sum_{1}^{\infty}\left|b_{n}\right|^{2} \leq C_{b}\|f\|^{2}$. Once again, $C_{\phi}$ is bounded.

We now show that the conditions on $\phi$ are necessary. We shall use the following facts (see $[\mathrm{GH}])$; "extension" will always mean "analytic extension".

FACT 1 ([GH] 1.2 p. 315). If $\phi(s)=c_{0} s+\varphi(s)$, with $c_{0} \in \mathbb{N}$ and $\varphi \in \mathcal{D}$, maps $\mathbb{C}_{\theta}$ to $\mathbb{C}_{\tau}$, and if $\phi_{\chi}(s)=c_{0} s+\varphi_{\chi}(s), \chi \in S$, then $\phi_{\chi}$ extends to a map: $\mathbb{C}_{\theta} \rightarrow \mathbb{C}_{\tau}$.

Just use the property that $\varphi_{\chi}(s)=\lim _{N \rightarrow \infty} \varphi\left(s+i t_{N}\right)$, for some sequence $\left(t_{N}\right)$ of real numbers.

FACT 2 ([GH] Prop. 4.3, p. 321). If $\phi$ is representable and maps $\mathbb{C}_{\theta}$ to $\mathbb{C}_{\frac{1}{2}}$, then $(f \circ \phi)_{\chi}(s)=f_{\chi} c_{0} \circ \phi_{\chi}(s), \forall f \in \mathcal{H}^{2}, \forall \chi \in S$.

FACT 3 ([GH] Prop. 5.1, p. 322). If $C_{\phi}: \mathcal{H}^{2} \rightarrow \mathcal{H}^{2}$ (with $\phi: \mathbb{C}_{\frac{1}{2}} \rightarrow \mathbb{C}_{\frac{1}{2}}$ ), then for almost every $\chi \in S, \phi_{\chi}$ has an analytic extension to $\mathbb{C}_{0}$.

We know that $\phi$ is representable by Theorem 4.1 ; for each integer $n \geq 2,\left(n^{-\phi}\right)_{\chi}$ can be almost surely extended to $\mathbb{C}_{0}$, by Helson's theorem 4.3 ; and we have $\left(n^{-\phi}\right)_{\chi}=$ $\chi(n)^{c_{0}} n^{-\phi_{\chi}}$. It follows that $\phi_{\chi}$ itself has almost surely an extension to $\mathbb{C}_{0}$.

Now, suppose first that $c_{0} \geq 1$. We know from Fact 3 that $\phi_{\chi}$ can almost surely be extended to $\mathbb{C}_{0}$, and the main point is that $\phi_{\chi}\left(\mathbb{C}_{0}\right) \subset \mathbb{C}_{0}$; if this were not so, we would find $s_{0} \in \mathbb{C}_{0}$ with $\Re \phi_{\chi}\left(s_{0}\right)=0$ and $\phi_{\chi}^{\prime}\left(s_{0}\right) \neq 0$, and Fact 2 would imply that the formula $f_{\chi} c_{0}=(f \circ \phi)_{\chi} \circ \phi_{\chi}^{-1}$ gives an extension of $f_{\chi} c_{0}$ across a small segment of the imaginary axis $\Re s=0$; since $f_{\chi}$ and $f_{\chi} c_{0}$ have the same measure distribution, we see that, for each $f \in \mathcal{H}^{2}$, the axis $\Re s=0$ is not a boundary for $f_{\chi}$, with positive probability; but if we take $f(s)=\sum_{p} \frac{1}{\sqrt{p} \log p} p^{-s} \in \mathcal{H}^{2}, p$ running over primes, the variables $\chi(p)$ are symmetric and independent, and the conclusion a) of Theorem 3.2 is contradicted. We thus have $\phi_{\chi}: \mathbb{C}_{0} \rightarrow \mathbb{C}_{0}$, and Fact 1 shows that $\phi=\left(\phi_{\chi}\right)_{\bar{\chi}}$ has an extension to $\mathbb{C}_{0}$, with $\phi\left(\mathbb{C}_{0}\right) \subset \mathbb{C}_{0}$.

If $c_{0}=0$, Fact 2 gives $(f \circ \phi)_{\chi}=f \circ \phi_{\chi}$, for any $f \in \mathcal{H}$. If $\phi_{\chi}$ does not map $\mathbb{C}_{0}$ to $\mathbb{C}_{\frac{1}{2}}$, the same reasoning shows that $f$ can be extended across a small segment of the axis $\Re s=\frac{1}{2}$. Now, take $f$ as in b) of Theorem 3.2 to get a contradiction.

Having seen that boundedness of composition operators $C_{\phi}$ on $\mathcal{H}^{2}$ is far from being automatic (although a complete description of the picture is available through Theorem 4.2), in contrast with the case of the Hardy space $H^{2}$, we now turn to the problem 
of compactness. Here, the situation is not yet fully understood, and to the best of my knowledge there are only two papers ([B2], [FQV]) giving partial answers to the question. One natural thing is to try to take advantage of the integral representation 23 to extend the result of J. Shapiro for $H^{2}$. This was partially done by Bayart [B2] who introduced the new counting function

$$
N_{\phi}(s)=\sum_{w \in \phi^{-1}(s)} \Re w \text { if } s \in \phi\left(\mathbb{C}_{0}\right), \quad N_{\phi}(s)=0 \text { otherwise. }
$$

This counting function seems to be appropriate when $c_{0} \geq 1$. In fact, we have Theorem 4.5 ([B2]). Let $\phi: \mathbb{C}_{0} \rightarrow \mathbb{C}_{0}, \phi(s)=c_{0} s+\varphi(s), c_{0} \geq 1$. Then

$$
N_{\phi}(s) \leq \frac{1}{c_{0}} \Re s \quad \text { for all } s \in \mathbb{C}_{0} \text {. }
$$

This is the analogue of Littlewood's inequality (14), where $\phi(0)=0$ becomes $\phi(\infty)=\infty$.

TheOREM 4.6 ([B2]). Let $\phi: \mathbb{C}_{0} \rightarrow \mathbb{C}_{0}, \phi(s)=c_{0} s+\varphi(s), c_{0} \geq 1$. Suppose that:

a) $\Im \varphi$ is bounded on $\mathbb{C}_{0}$;

b) $N_{\phi}(s)=o(\Re s)$ if $\Re s \stackrel{>}{\longrightarrow}$.

Then, $C_{\phi}$ is compact on $\mathcal{H}^{2}$.

We do not know whether the converse is true; the equivalent of a) in Theorem 1.4 becomes (using the properties of the reproducing kernel of $\mathcal{H}^{2}$ ):

$$
\text { If } C_{\phi}: \mathcal{H}^{2} \rightarrow \mathcal{H}^{2} \text { is compact, } \Re \phi(a)>\frac{1}{2} \text { for } \Re s=\frac{1}{2} .
$$

But this has no interest: if $\phi(s) \neq s+i \tau$, one can show that $\phi\left(\overline{\mathbb{C}_{1 / 2}}\right) \subset \overline{\mathbb{C}_{\frac{1}{2}+\varepsilon}}$ for some $\varepsilon>0$, and if $\phi(s)=s+i \tau, C_{\phi}$ is clearly not compact! At the end of this section, we will restrict ourselves to symbols $\phi: \mathbb{C}_{1 / 2} \rightarrow \mathbb{C}_{1 / 2}$ of the following type:

$$
\phi(s)=c_{0} s+c_{1}+\sum_{j=1}^{d} c_{q_{j}} q_{j}^{-s}, \quad c_{q_{j}} \neq 0,
$$

where $2 \leq q_{1}<\ldots<q_{d}$ are "multiplicatively independent", i.e. each integer $n$ can be written as $n=q_{1}^{\alpha_{1}} \ldots q_{d}^{\alpha_{d}}, \alpha_{j} \in \mathbb{N}$, in at most one way (e.g. $q_{1}=2, q_{2}=6, q_{3}=30$ ).

In that case, the real numbers $\log q_{1}, \ldots, \log q_{d}$ are rationally independent, and the Kronecker approximation theorem implies that

$$
\inf _{t} \Re \phi(\sigma+i t)=c_{0} \sigma+\Re c_{1}-\sum_{j=1}^{d}\left|c_{q_{j}}\right| q_{j}^{-\sigma}, \quad \text { for each } \sigma>0 .
$$

So that the boundedness condition of Theorem 4.2 reads:

$$
\begin{gathered}
\text { If } c_{0} \geq 1, C_{\phi} \text { is bounded iff } \Re c_{1} \geq\left|c_{q_{1}}\right|+\cdots+\left|c_{q_{d}}\right| . \\
\text { If } c_{0}=0, C_{\phi} \text { is bounded iff } \Re c_{1} \geq \frac{1}{2}+\left|c_{q_{1}}\right|+\cdots+\left|c_{q_{d}}\right| .
\end{gathered}
$$

A natural guess would be that $C_{\phi}$ is compact if and only if the inequalities in (29) and (30) are strict; this is not quite the case, and big differences between the cases $c_{0} \geq 1$, $c_{0}=0$ appear; we have the following results: 
THEOREM 4.7 ([B2]). Suppose that $c_{0} \geq 1$. Then, the following are equivalent:

a) $\Re c_{1}>\left|c_{q_{1}}\right|+\cdots+\left|c_{q_{d}}\right|$;

d) $\phi\left(\mathbb{C}_{0}\right) \subset \mathbb{C}_{\varepsilon}$ for some $\varepsilon>0$;

c) $C_{\phi}$ is compact.

One only has to prove that $\mathrm{c}) \Rightarrow \mathrm{a}$ ). Improving the useless $(*)$, one shows that the compactness of $C_{\phi}$ implies: $\lim _{\Re s \rightarrow 0} \frac{\Re \phi(s)}{\Re s}=+\infty$, and this easily implies a).

If $c_{0}=0$, the integer $d$ plays a crucial role, comparable to the role which it plays in a famous theorem of Pólya: let $e_{1}, \ldots, e_{d}$ be the canonical basis of $\mathbb{R}^{d}, X$ be a random variable such that $P\left(X= \pm e_{j}\right)=\frac{1}{2 d}, 1 \leq j \leq d$, and $\left(S_{n}\right)$ be the random walk on $\mathbb{Z}^{d}$ associated to $X$, i.e. $S_{n}=X_{1}+\cdots+X_{n}$, where the $X_{n}^{\prime} s$ are independent copies of $X$; then

THEOREM 4.8 ([Re]). Let $\left(S_{n}\right)$ be the above random walk on $\mathbb{Z}^{d}$. Then:

a) If $d=1$ or $2,\left(S_{n}\right)$ is almost surely recurrent, i.e. $\underline{\lim }\left\|S_{n}\right\|=0$ a.s.

b) If $d \geq 3,\left(S_{n}\right)$ is almost surely transient, i.e. $\underline{\lim }\left\|S_{n}\right\|=\infty$ a.s.

In fact, $\sum P\left(S_{n}=0\right)=\infty$ iff $d \leq 2$. The analogue here will be the series $\sum \| C_{\phi}\left(n^{-s} \|^{2}\right.$ and the Hilbert-Schmidt character of $C_{\phi}$ according to the values of $d$. We have more precisely the following statements:

THEOREM 4.9 ([FQV]). Suppose that $d=1$ and $c_{0}=0$. Then, the following are equivalent:

a) $\Re c_{1}>\left|c_{q_{1}}\right|+\frac{1}{2}$

b) $\phi\left(\mathbb{C}_{0}\right) \subset \mathbb{C}_{\varepsilon+\frac{1}{2}}$ for some $\varepsilon>0$;

c) $C_{\phi}$ is compact;

d) $C_{\phi}$ is Hilbert-Schmidt.

By analyzing $C_{\phi} C_{\phi}^{*}$, if $\Re c_{1}=\left|c_{q_{1}}\right|+\frac{1}{2}$ one is led to the equivalent study of $C: H^{2} \rightarrow$ $H^{2}$ given by

$$
C\left(z^{i}\right)=\sum_{j \geq 0} \frac{(i+j) !}{i ! j !} 2^{-i-j} z^{j},
$$

and we see that $C=M C_{h}$, where $M: H^{2} \rightarrow H^{2}$ is the multiplication operator by the $H^{\infty}$-function

$$
(1-z / 2)^{-1},
$$

and $h: D \rightarrow D$ is given by $h(z)=\frac{1}{2-z}$. We have $\lim _{r \leq 1} \frac{1-h(r)}{1-r}=1<\infty$, therefore (see Theorem 1.4) $C_{h}$ and hence $C$ are not compact; the assertion d) will be proved later.

TheOREM 4.10 ([FQV]). Suppose that $d=2$ and $c_{0}=0$. Then:

a) $C_{\phi}$ is always compact;

b) $C_{\phi}$ is Hilbert-Schmidt if and only if $\Re c_{1}>\frac{1}{2}+\left|c_{q_{1}}\right|+\left|c_{q_{2}}\right|$;

c) There are composition operators on $\mathcal{H}^{2}$ which are compact and not Hilbert-Schmidt. 
The most difficult point is a); by analyzing $C_{\phi} C_{\phi}^{*}$, if $\Re c_{1}=\frac{1}{2}+\left|c_{q_{1}}\right|+\left|c_{q_{2}}\right|$, one is led to the equivalent study of an operator $C: H^{2}\left(\mathbb{T}^{2}\right) \rightarrow H^{2}\left(\mathbb{T}^{2}\right)$ which is no longer a composition operator as in Theorem 4.9, but the operator $f \mapsto \int C(u, v ; x, y) f(u, v) d u d v$ associated with the kernel

$$
C(u, v ; x, y)=\frac{1}{4-a\left(e^{i x}+e^{-i u}\right)-b\left(e^{i y}+e^{-i v}\right)}
$$

on $\mathbb{T}^{4}$, with $a, b>0$ and $a+b=2$. One shows that $C \in L^{2}\left(\mathbb{T}^{4}\right)$, so that the associated operator $C$ is Hilbert-Schmidt, which implies that $C_{\phi}$ is in the Schatten class $S_{4}$, and a fortiori is compact; b) is proved later; an example for c) is provided by $\phi(s)=\frac{3}{2}+\frac{2^{-s}+3^{-s}}{2}$; it is interesting to compare with b) of corollary 1.5 .

THEOREM 4.11 ([FQV]). Suppose that $d \geq 3$. Then

a) If $c_{0} \geq 1, C_{\phi}$ is Hilbert-Schmidt if and only if $\Re c_{1} \geq \frac{1}{2}+\sum_{j=1}^{d}\left|c_{q_{j}}\right|$.

b) If $c_{0}=0, C_{\phi}$ is Hilbert-Schmidt if and only if $C_{\phi}$ is bounded. In particular, there are composition operators $C_{\phi}$ on $\mathcal{H}^{2}$, with $c_{0}=0$ and $\inf _{\Re s>0} \Re \phi(s)=\frac{1}{2}$, which are Hilbert-Schmidt.

The proof uses the following:

LEMMA 4.12. Let $d$ be an integer $\geq, \delta_{1}, \ldots, \delta_{d}>0, n \geq 1$, and

$$
S_{n}=\sum_{i_{1}+\cdots+i_{d}=n}\left(\frac{n !}{i_{1} ! \ldots i_{d} !}\right)^{2} \delta_{1}^{2 i_{1}} \ldots \delta_{d}^{2 i_{d}}
$$

be the sum of the squares of the multinomial coefficients. Then, as $n \rightarrow \infty$ :

$$
S_{n} \sim \lambda n^{-\frac{d-1}{2}}\left(\delta_{1}+\cdots+\delta_{d}\right)^{2 n},
$$

where $\lambda>0$ is a constant independent from $n$.

Proof. For equal $\delta_{j}$ 's, this is nothing but Pólya's theorem, and the general case is similar; we can assume $\delta_{1}+\cdots+\delta_{d}=1$; denote by $Q_{d}$ the unit cube $\left[-\frac{1}{2}, \frac{1}{2}\right]^{d}$ of $\mathbb{R}^{d}$ and set $e(x)=e^{2 i \pi x}, c(x)=\cos 2 \pi x, s(x)=\sin \pi x$. Parseval's relation gives

$$
S_{n}=\int_{Q_{d}}\left|\delta_{1} e\left(\theta_{1}\right)+\cdots+\delta_{d} e\left(\theta_{d}\right)\right|^{2 n} d \theta_{1} \ldots d \theta_{d} .
$$

Now, we have

$$
\begin{aligned}
\left|\sum_{j=1}^{d} \delta_{j} e\left(\theta_{j}\right)\right|^{2} & =\sum_{j=1}^{d} \delta_{j}^{2}+2 \sum_{1 \leq j<k \leq d} \delta_{j} \delta_{k} c\left(\theta_{j}-\theta_{k}\right) \\
& =\sum_{j=1}^{d} \delta_{j}^{2}+2 \sum_{1 \leq j<k \leq d} \delta_{j} \delta_{k}-2 \sum_{1 \leq j<k \leq d} \delta_{j} \delta_{k}\left(1-c\left(\theta_{j}-\theta_{k}\right)\right) \\
& =1-4 \sum_{1 \leq j<k \leq d} \delta_{j} \delta_{k} s^{2}\left(\theta_{j}-\theta_{k}\right) .
\end{aligned}
$$


Make the change of variable $\theta_{k}-\theta_{1}=\varphi_{k}, 2 \leq k \leq d$, and use the translation-invariance to get

$$
\begin{aligned}
& S_{n}=\int_{Q_{d-1}}\left(1-4 \sum_{2 \leq k \leq d} \delta_{1} \delta_{k} s^{2}\left(\varphi_{k}\right)-4 \sum_{2 \leq j<k \leq d} \delta_{j} \delta_{k} s^{2}\left(\varphi_{j}-\varphi_{k}\right)\right)^{n} d \varphi_{2} \ldots d \varphi_{d} \\
= & n^{-\frac{d-1}{2}} \int_{\sqrt{n} Q_{d-1}}\left(1-4 \sum_{2 \leq k \leq d} \delta_{1} \delta_{k} s^{2}\left(\frac{\Psi_{k}}{\sqrt{n}}\right)-4 \sum \delta_{j} \delta_{k} s^{2}\left(\frac{\Psi_{j}-\Psi_{k}}{\sqrt{n}}\right)\right)^{n} d \Psi_{2} \ldots d \Psi_{d} \\
= & \quad\left(\text { where } \sum \text { is an abbreviation for } \sum_{2 \leq j<k \leq d}\right) \\
& \int_{\mathbb{R}^{-\frac{d-1}{2}}} f_{n}\left(\Psi_{2}, \ldots, \Psi_{d}\right) d \Psi_{2} \ldots d \Psi_{d},
\end{aligned}
$$

where we have

$$
\begin{array}{r}
0 \leq f_{n}\left(\Psi_{2}, \ldots, \Psi_{d}\right) \leq \exp \left(-4 n \delta_{1} \sum_{k=2}^{d} \delta_{k} s^{2}\left(\frac{\Psi_{k}}{\sqrt{n}}\right)\right) 1_{\sqrt{n} Q_{d-1}}\left(\Psi_{2}, \ldots, \Psi_{d}\right) \\
\leq \exp \left(-16 \sum_{k=2}^{d} \delta_{1} \delta_{k} \Psi_{k}^{2}\right), \quad \text { because }|s(x)| \geq 2|x| \text { for }|x| \leq \frac{1}{2} .
\end{array}
$$

Moreover, $f_{n}\left(\Psi_{2}, \ldots, \Psi_{d}\right) \rightarrow g\left(\Psi_{2}, \ldots, \Psi_{d}\right)$ as $n \rightarrow \infty$, with

$$
g\left(\Psi_{2}, \ldots, \Psi_{d}\right)=\exp \left(-4 \sum_{k=2}^{d} \delta_{1} \delta_{k} \Psi_{k}^{2}-4 \sum_{2 \leq j<k \leq d} \delta_{j} \delta_{k}\left(\Psi_{j}-\Psi_{k}\right)^{2}\right) .
$$

Lebesgue's dominated convergence theorem now shows that $S_{n} \sim \lambda n^{-\frac{d-1}{2}}$, with $\lambda=$ $\int_{\mathbb{R}^{d-1}} g\left(\Psi_{2}, \ldots, \Psi_{d}\right) d \Psi_{2} \ldots d \Psi_{d}>0$

It is now easy to complete the proofs of Theorems $4.9,4.10,4.11$ with the help of the following simple lemma:

Lemma 4.13. Let $b>1$ be fixed, and let $a>0$ tend to infinity. Then

$$
\sum_{k \geq 1} \frac{(\log k)^{a}}{k^{b}} \sim \int_{1}^{\infty} \frac{(\log t)^{a}}{t^{b}} d t=\frac{\Gamma(a+1)}{(b-1)^{a+1}}
$$

where $\Gamma$ is the Euler gamma function.

Regardless of the value of $c_{0}$, and using the independence of the $q_{j}$ 's, we get

$$
\sum_{n=1}^{\infty}\left\|C_{\phi}\left(n^{-s}\right)\right\|^{2}=\sum_{i_{1}, \ldots, i_{d} \geq 0} \frac{\delta_{1}^{2_{i_{1}}} \ldots \delta_{d}^{2_{i}}}{\left(i_{1} ! \ldots i_{d} !\right)^{2}} \sum_{n=1}^{\infty} \frac{(\log n)^{2\left(i_{1}+\cdots+i_{d}\right)}}{n^{2 \gamma_{1}}}
$$

where $\gamma_{1}=\Re c_{1}$ and $\delta_{j}=\left|c_{q_{j}}\right|$. So that, using Lemmas 4.12 and 4.13 , we get with obvious notations: 


$$
\begin{aligned}
\sum_{n=1}^{\infty}\left\|C_{\phi}\left(n^{-s}\right)\right\|^{2} & \approx \sum_{i_{1}, \ldots, i_{d} \geq 0} \frac{\delta_{1}^{2_{i_{1}}} \ldots \delta_{d}^{2_{i_{d}}}}{\left(i_{1} ! \ldots i_{d} !\right)^{2}} \frac{\left(2 i_{1}+\cdots+2 i_{d}\right) !}{\left(2 \gamma_{1}-1\right)^{2\left(i_{1}+\cdots+i_{d}\right)}} \\
& =\sum_{l=1}^{\infty} \frac{(2 l) !}{\left(2 \gamma_{1}-1\right)^{2 l}(l !)^{2}} \sum_{i_{1}+\ldots+i_{d}=l}\left(\frac{l !}{i_{1} ! \ldots i_{d} !}\right)^{2} \delta_{1}^{2 i_{1}} \ldots \delta_{d}^{2 i_{d}} \\
& =\sum_{l=1}^{\infty} \frac{C_{2 l}^{l}}{\left(2 \gamma_{1}-1\right)^{2 l}} S_{l} \approx \sum_{l=1}^{\infty} \frac{4^{l}}{\sqrt{l}\left(2 \gamma_{1}-1\right)^{2 l}} \frac{1}{l^{\frac{d-1}{2}}}\left(\delta_{1}+\ldots+\delta_{d}\right)^{2 l} \\
& =\sum_{l=1}^{\infty} l^{-d / 2}\left(\frac{2\left(\delta_{1}+\ldots+\delta_{d}\right)}{2 \gamma_{1}-1}\right)^{2 l} .
\end{aligned}
$$

Now, if $d=1$ or 2 , the last series converges if and only if $2 \gamma_{1}-1>2\left(\delta_{1}+\ldots+\delta_{d}\right)$, while if $d \geq 3$, it converges if and only if $2 \gamma_{1}-1 \geq 2\left(\delta_{1}+\ldots+\delta_{d}\right)$. This finishes the proof.

Let us mention (see $[\mathrm{FQV}]$ ) that Theorem 4.11 holds for more general symbols $\phi(s)=$ $c_{0} s+c_{1}+\sum_{j=1}^{\infty} c_{q_{j}} q_{j}^{-s}$, where $\sum\left|c_{q_{j}}\right|<\infty, c_{q_{j}} \neq 0$, and at least three of the $q_{j}$ 's are independent; $C_{\phi}$ is Hilbert-Schmidt as soon as it is bounded. Nevertheless, the general picture for compactness is not clear, in spite of Theorem 4.6, all the more as $\phi$ is never injective if $c_{0}=0[\mathrm{Fa}]$. We hope to come back to this problem of compactness in another work.

In contrast with the case of the unit disk, there are very few invertible or isometric composition operators on $\mathcal{H}^{2}$. Indeed we have [B1]:

THEOREM 4.14. Let $C_{\phi}: \mathcal{H}^{2} \rightarrow \mathcal{H}^{2}$ be bounded. Then, the following are equivalent:

a) $C_{\phi}$ is invertible;

b) $C_{\phi}$ is Fredholm;

c) $\phi(s)=s+i \tau$, where $\tau \in \mathbb{R}$.

Theorem 4.15. Let $C_{\phi}: \mathcal{H}^{2} \rightarrow \mathcal{H}^{2}$ be bounded, with $\phi(s)=c_{0} s+\varphi(s)$. Assume that the Dirichlet series of $\varphi$ converges uniformly on $\mathbb{C}_{0}$. Then, the following are equivalent:

a) $C_{\phi}$ is isometric;

b) $C_{\phi}$ is similar to an isometry;

c) $\phi(s)=c_{0} s+i \tau$, where $c_{0} \geq 1$ and $\tau \in \mathbb{R}$.

5. The Wiener-Dirichlet algebra $\mathcal{A}^{+}$. This is the Banach algebra of Dirichlet series $f(s)=\sum_{1}^{\infty} a_{n} n^{-s}$, with $\|f\|=\sum_{1}^{\infty}\left|a_{n}\right|<\infty$. This algebra is commutative and unital, and can be interpreted as a space of analytic functions on $\mathbb{C}_{0}$; as we already mentioned in Section 4, the study of function spaces formed by Dirichlet series has been the subject of some recent interest (see [B1], [B2], [FQ], [FQV], [HLS], [GH], [McCa] for example). Now, a method due to Bohr (see [Q2] for other applications) identifies the algebra $\mathcal{A}^{+}$with the algebra $A^{+}\left(\mathbb{T}^{\infty}\right)$ formed by absolutely convergent Taylor series in countably many variables (this allows one to identify the spectrum of $\mathcal{A}^{+}$as $\bar{D}^{\infty}$ ). Let us recall the way this identification is carried out. Let $\left(p_{j}\right)_{j \geq 1}$ be the increasing sequence of prime numbers 
$\left(p_{1}=2, p_{2}=3, \ldots\right)$. A function $f$ in $A^{+}\left(\mathbb{T}^{\infty}\right)$ can be written as

$$
f(z)=\sum_{\alpha \in \mathbb{N}_{0}^{(\infty)}} a_{\alpha} z^{\alpha}, \quad \text { with }\|f\|_{A^{+}\left(\mathbb{T}^{\infty}\right)}=\sum_{\alpha}\left|a_{\alpha}\right|<\infty,
$$

where, as usual, we set $\alpha=\left(\alpha_{1}, \ldots, \alpha_{r}, 0,0, \ldots\right)$ and $z^{\alpha}=z_{1}^{\alpha_{1}} \ldots z_{r}^{\alpha_{r}}$ for $z=\left(z_{j}\right)_{j \geq 1}$. Then $\Delta: \mathcal{A}^{+} \rightarrow A^{+}\left(\mathbb{T}^{\infty}\right)$ is defined by

$$
\Delta\left(\sum_{n=1}^{\infty} a_{n} n^{-s}\right)=\sum_{n=1}^{\infty} a_{n} z_{1}^{\alpha_{1}} \ldots z_{r}^{\alpha_{r}}, \quad n=p_{1}^{\alpha_{1}} \ldots p_{r}^{\alpha_{r}} .
$$

$\Delta$ is an isometric isomorphism; for $s \in \mathbb{C}_{0}, f \in \mathcal{A}^{+}, g \in A^{+}\left(\mathbb{T}^{\infty}\right)$, we set

$$
z^{[s]}=\left(p_{j}^{-s}\right)_{j \geq 1} \in D^{\infty} ; \quad\|f\|_{\infty}=\sup _{s \in \mathbb{C}_{0}}|f(s)| ;\|g\|_{\infty}=\sup _{z \in D^{\infty}}|g(z)| .
$$

With those notations, $\Delta$ has the two following properties (see [B2]):

$$
\begin{gathered}
\Delta f\left(z^{[s]}\right)=f(s), \quad \text { for any } f \in \mathcal{A}^{+} \text {and any } s \in \mathbb{C}_{0} ; \\
\|\Delta f\|_{\infty}=\|f\|_{\infty}, \quad \text { for each } f \in \mathcal{A}^{+} .
\end{gathered}
$$

When we study composition operators $C_{\phi}: \mathcal{A}^{+} \rightarrow \mathcal{A}^{+}$associated with an analytic $\phi: \mathbb{C}_{0} \rightarrow \mathbb{C}_{0}$, Theorem 4.1 indicates that $\phi$ has to be representable, which we assume once and for all in the sequel (as well as the fact that $\phi$ is non-constant, to avoid trivialities). A big difference with the case of the Wiener algebra at once appears: in the latter case, the identity function $z \in A^{+}$, so that if we want $C_{\phi}$ to map $A^{+}$to itself, we have to assume that $\phi$ itself belongs to $A^{+}$; here, the identity function $s$ does not belong to $\mathcal{A}^{+}$, and in fact we do not know if the condition $\phi \in \mathcal{A}^{+}$is necessary for $C_{\phi}$ to map $\mathcal{A}^{+}$to itself; we have the following Theorems 5.1 and 5.2, which may be viewed as analogues of Theorems 2.1 and 2.2 respectively, with $\phi^{n}$ being replaced by $n^{-\phi}$ (see [BFLQ1]):

Theorem 5.1. Let $\phi: \mathbb{C}_{0} \rightarrow \mathbb{C}_{0}$. Then

a) $C_{\phi}$ maps $\mathcal{A}^{+}$to itself if and only if $n^{-\phi} \in \mathcal{A}^{+}$for $n \in \mathbb{N}$, and $\left\|n^{-\phi}\right\| \leq C$ for each $n \in \mathbb{N}$.

b) If $\phi(s)=c_{0} s+\sum_{1}^{\infty} c_{n} n^{-s}$ with $\sum_{1}^{\infty}\left|c_{n}\right|<\infty$, then $C_{\phi}$ maps $\mathcal{A}^{+}$to itself as soon as $\Re c_{1} \geq \sum_{2}^{\infty}\left|c_{n}\right|$, regardless of the value of $c_{0}$.

Theorem 5.2. Let $\phi: \mathbb{C}_{0} \rightarrow \mathbb{C}_{0}$. Then

a) $C_{\phi}: \mathcal{A}^{+} \rightarrow \mathcal{A}^{+}$is compact if and only if $\left\|n^{-\phi}\right\| \rightarrow 0$ as $n \rightarrow \infty$.

b) If $C_{\phi}$ is compact, we have $\phi\left(\mathbb{C}_{0}\right) \subset \mathbb{C}_{\delta}$ for some $\delta>0$. The converse is true if $\phi(s)=c_{0} s+\varphi(s)$, with $\varphi \in \mathcal{A}^{+}$.

a) is proved exactly as Theorem 2.2. The existence of $\delta$ in b) is easy: set $\delta=$ $\inf _{s \in \mathbb{C}_{0}} \Re \phi(s)$; we have $n^{-\delta}=\left\|n^{-\phi}\right\|_{\infty} \leq\left\|n^{-\phi}\right\|$, so $n^{-\delta} \rightarrow 0$ by a), and $\delta$ is positive; conversely, if $\delta>0$ and $\varphi \in \mathcal{A}^{+}$, we use the following facts:

FACT 1. If $\phi(s)=c_{0} s+\varphi(s)$ maps $\mathbb{C}_{\theta}$ to $\mathbb{C}_{\tau}$ and $\varphi$ is non-constant, $\varphi$ maps $\mathbb{C}_{\theta}$ to $\mathbb{C}_{\tau-c_{0} \theta}([\mathrm{GH}])$.

FACT 2. If $v \in \mathcal{A}^{+}$and $r \geq 1$, then $r^{-v} \in \mathcal{A}^{+}$, and $\left\|r^{-v}\right\| \leq r\|v\|$. 
This is a simple and well-known property of the norm in a Banach algebra. Now, let $\phi(s)=c_{0} s+\varphi(s)$, with $\phi\left(\mathbb{C}_{0}\right) \subset \mathbb{C}_{\delta}$ and $\varphi \in \mathcal{A}^{+}$. We have $\varphi\left(\mathbb{C}_{0}\right) \subset \mathbb{C}_{\delta}$ by Fact 1 , and if $\Psi=2^{-\varphi}$, the spectral radius formula and Bohr's method (see the beginning of this section) give

$$
\lim _{j \rightarrow \infty}\left\|\Psi^{j}\right\|^{1 / j}=\sup _{h \in S p \mathcal{A}^{+}}|h(\Psi)|=\sup _{s \in \mathbb{C}_{0}}|\Psi(s)| \leq 2^{-\delta},
$$

and in particular $\left\|\Psi^{j}\right\| \rightarrow 0$; any integer $n \geq 2$ can be written as $n=2^{j} r, j \in \mathbb{N}$, $1 \leq r \leq 2$, so that

$$
\left\|n^{-\phi}\right\|=\left\|n^{-\varphi}\right\|=\left\|2^{-j \varphi} r^{-\varphi}\right\| \leq\left\|\Psi^{j}\right\|\left\|r^{-\varphi}\right\| \leq\left\|\Psi^{j}\right\| 2^{\|\varphi\|},
$$

using Fact 2 and $r \leq 2$. Therefore, $\left\|n^{-\phi}\right\| \rightarrow 0$, and $C_{\phi}$ is compact by Part a) of the Theorem.

Note that the assumption $\phi\left(\mathbb{C}_{0}\right) \subset \mathbb{C}_{\delta}$ holds if $\Re c_{1}>\sum_{2}^{\infty}\left|c_{n}\right|$.

However, conditions like $\sum_{2}^{\infty}\left|c_{n}\right| \leq \Re c_{1}$ (or $<\Re c_{1}$ ) are not necessary to have boundedness or compactness, as shown by the following examples,

THEOREM 5.3. Let $\phi(s)=c_{0} s+c_{1}+c_{r} r^{-s}+c_{r^{2}} r^{-2 s}$, where $r \geq 2$, and $c_{r}, c_{r^{2}}>0$.

a) If

$$
\Re c_{1}>\frac{\left(c_{r}\right)^{2}}{8 c_{r^{2}}}+c_{r^{2}}
$$

then $C_{\phi}: \mathcal{A}^{+} \rightarrow \mathcal{A}^{+}$is compact.

b) If $C_{\phi}: \mathcal{A}^{+} \rightarrow \mathcal{A}^{+}$is bounded (resp. compact) and moreover $c_{r} \leq 4 c_{r^{2}}$, we must have $\Re c_{1} \geq \frac{\left(c_{r}\right)^{2}}{8 c_{r^{2}}}+c_{r^{2}}$ (resp. we must have (35)).

c) If $\Re c_{1}=\frac{\left(c_{r}\right)^{2}}{8 c_{r^{2}}}+c_{r^{2}}$, then $C_{\phi}$ maps $\mathcal{A}^{+}$to itself if and only if $c_{r} \neq 4 c_{r^{2}}$.

Proof. W.l.o.g., we assume $r=2$. It is easy to check that

$$
\text { If } c_{2} \leq 4 c_{4} \text { (resp. }>4 c_{4} \text { ), we have } \inf _{s \in \mathbb{C}_{0}} \Re \phi(s)=\Re c_{1}-\frac{c_{2}^{2}}{8 c_{4}}-c_{4}
$$

(resp. inf $\ldots=\Re c_{1}+c_{4}-c_{2}$ ). Therefore, a) and b) follow from Theorem 5.2, since $\frac{c_{2}^{2}}{8 c_{4}}+c_{4}>c_{2}-c_{4}$ if $c_{2}>4 c_{4}$. But, one may give a more informative proof, which has other applications (see Section 6).

Let $H_{0}, H_{1}, \ldots$ be the sequence of Hermite polynomials, whose generating function is

$$
\sum_{k=0}^{\infty} \frac{H_{k}(\lambda)}{k !} x^{k}=\exp \left(2 \lambda x-x^{2}\right) .
$$

It is possible to show [BFLQ1] that putting $\alpha=\Re c_{1}-\frac{c_{2}^{2}}{8 c_{4}}-c_{4}$, we have:

$$
\begin{gathered}
\sum_{k=0}^{\infty} \frac{\left|H_{k}(\lambda)\right|}{k !} x^{k} \leq C(1+x)^{1 / 2} \exp \left(x^{2}+\lambda^{2} / 2\right), \quad \text { where } C \text { is a constant; } \\
\left\|n^{-\phi}\right\|=n^{-\Re c_{1}} \sum_{k=0}^{\infty} \frac{\left|H_{k}\left(\lambda_{n}\right)\right|}{k !} x_{n}^{k} \leq C(\log n)^{1 / 4} n^{-\alpha}
\end{gathered}
$$


where $x_{n}=\sqrt{c_{4} \log n}$, and $\lambda_{n}=-\frac{-c_{2}}{2 \sqrt{c_{4}}} \sqrt{\log n}$;

$$
\text { If } c_{2} \leq 4 c_{4},\left\|n^{-\phi}\right\| \geq n^{-\alpha} \text { for } n \geq 1 \text {. }
$$

And those estimates clearly give an alternative proof of a) and b).

For c), if $c_{2}>4 c_{4}$, we have $\inf _{s \in \mathbb{C}_{0}} \Re \phi(s)>0$ by (36), and we are done. If $c_{2} \leq 4 c_{4}$, we have

$$
\left\|2^{-j \phi}\right\|_{\mathcal{A}^{+}}=\left\|\Psi^{j}\right\|_{A^{+}(\mathbb{T})},
$$

where $\Psi(z)=\exp \left[-\left(c_{1}+c_{2} z+c_{4} z^{2}\right) \log 2\right]$, and $\|\Psi\|_{\infty}=1$.

We then apply to $\Psi$ the Newman criterion (15) on ordinary points: if $\left|\psi\left(e^{i \theta_{0}}\right)\right|=1$, the coefficient $\alpha_{2}$ of $t^{2}$ in the Taylor expansion of $\log \Psi\left(e^{i\left(\theta_{0}+t\right)}\right)$ is $\alpha_{2}=\frac{c_{2}}{2} e^{i \theta_{0}}+2 c_{4} e^{2 i \theta_{0}}$, so that

$$
\Re \alpha_{2}=\frac{c_{2}}{2} \cos \theta_{0}+2 c_{4}\left(2 \cos ^{2} \theta_{0}-1\right) .
$$

Now, since $\Re c_{1}=\frac{c_{2}^{2}}{8 c_{4}}+c_{4}$, one easily checks that $\left|\Psi\left(e^{i \theta_{0}}\right)\right|=1$ implies $\cos \theta_{0}=\frac{-c_{2}}{4 c_{4}}$, so that

$$
\Re \alpha_{2}=\frac{c_{2}^{2}}{8 c_{4}}-2 c_{4} .
$$

Now, if $c_{2} \neq 4 c_{4}, \Re \alpha_{2} \neq 0, \theta_{0}$ is an ordinary point, $\left\|\Psi^{j}\right\|_{A^{+}}$is bounded, $\left\|2^{-j \phi}\right\|$ and therefore $\left\|n^{-\phi}\right\|$ (see the proof of Theorem 5.2) are bounded, and $C_{\phi}$ itself is bounded. If $c_{2}=4 c_{4}$, we have $\theta_{0}=\pi(\bmod 2 \pi), \log \Psi\left(e^{i\left(\theta_{0}+t\right)}\right)=d_{0}+d_{1} t+0 \cdot t^{2}+\alpha_{3} t^{3}+\ldots$, with $\alpha_{3}=(i \log 2) \frac{2 c_{4}}{3} \neq 0$ and $\Re \alpha_{3}=0$, so that $\theta_{0}$ is not an ordinary point, and $\left\|2^{-j \phi}\right\|$, $\left\|n^{-\phi}\right\|$ are not bounded. In particular, $\phi(s)=i a+c\left(3+4.2^{-s}+4^{-s}\right)$, with $a \in \mathbb{R}$ and $c>0$, provides an example of a symbol $\phi$ such that $\sum\left|c_{n}\right|<\infty$ and $\phi\left(\mathbb{C}_{0}\right) \subset \mathbb{C}_{0}$, but $C_{\phi}$ is not bounded on $\mathcal{A}^{+}$.

This shows that the situation as concerns boundedness and compactness is not yet fully understood. Let us now turn to the automorphisms and isometries; we first study the case of the algebras $A^{+}\left(\mathbb{T}^{k}\right), 1 \leq k<\infty$, or $A^{+}\left(\mathbb{T}^{\infty}\right)$.

TheOREM 5.4 ([BFLQ1]). Assume that the map $\phi: D^{k} \rightarrow \bar{D}^{k}$ induces a bounded operator $C_{\phi}: A^{+}\left(\mathbb{T}^{k}\right) \rightarrow A^{+}\left(\mathbb{T}^{k}\right)$. Then, $C_{\phi}$ is an automorphism if and only if $\phi(z)=$ $\left(\varepsilon_{1} z_{\sigma(1)}, \ldots, \varepsilon_{k} z_{\sigma(k)}\right)$ for some permutation $\sigma$ of $\{1, \ldots, k\}$ and some complex signs $\varepsilon_{1}, \ldots, \varepsilon_{k}$.

Proof. Suppose that $C_{\phi}$ is an automorphism; $\phi_{j}=C_{\phi}\left(z_{j}\right) \in A^{+}\left(\mathbb{T}^{k}\right)$, for $1 \leq j \leq k$, therefore $\phi$ has a continuous extension $\phi: \bar{D}^{k} \rightarrow \bar{D}^{k} ; C_{\phi}$ being surjective, $\phi$ is injective; Osgood's Theorem [Na] implies that det $\phi^{\prime}(z) \neq 0 \forall z \in D^{k}$, therefore $\phi$ is an open mapping on $D^{k}$, and $\phi\left(D^{k}\right) \subset D^{k}$; it is easy to check that $\phi: \bar{D}^{k} \rightarrow \bar{D}^{k}$ is onto, therefore $\phi\left(D^{k}\right)=D^{k}$, and $\phi$ is an analytic automorphism of $D^{k}$. Therefore, we know that [Na] $\phi$ separates the variables:

$$
\phi(z)=\left(\varepsilon_{j} \frac{z_{\sigma(j)}-a_{j}}{1-\bar{a}_{j} z_{\sigma(j)}}\right)_{1 \leq j \leq k}
$$

where $a_{j} \in D,\left|\varepsilon_{j}\right|=1$, and $\sigma$ is a permutation of $\{1, \ldots, k\}$. 
Now,

$$
\left\|\left(\varepsilon_{j}\left(\frac{z-a_{j}}{1-\bar{a}_{j} z}\right)\right)^{n}\right\|_{A^{+}}=\left\|\phi_{j}^{n}\right\|_{A^{+}\left(\mathbb{T}^{k}\right)}=O(1),
$$

and this implies, as in the proof of Theorem 2.3, that $a_{j}=0$, which finishes the proof.

We conjecture that the same result holds for $A^{+}\left(\mathbb{T}^{\infty}\right)$, but we are only able to prove the following (which will yet be sufficient for the case of $\mathcal{A}^{+}$): it is convenient to denote by $B=D^{\infty} \cap c_{0}$ the open unit ball of the Banach space $c_{0}$; then we have:

Theorem 5.5. Let $\phi=\left(\phi_{j}\right)_{j}: B \rightarrow B$ be an analytic map such that $C_{\phi}$ maps $A^{+}\left(\mathbb{T}^{\infty}\right)$ to itself. Assume that $C_{\phi}$ is an automorphism, and that moreover we have for each $k$ : $\phi_{k}(z)=z_{k}^{d_{k}} u_{k}(z)$, where $d_{k}$ is a integer $\geq 1$ and $u_{k}(0) \neq 0$; then, $\phi(z)=\left(\varepsilon_{j} z_{j}\right)_{j}$, for some sequence of complex signs $\varepsilon_{j}$.

Proof (see [BFLQ1]). Let $K=\bar{D}^{\infty} ; \phi$ is a homeomorphism of $K$, and the assumptions allow us to show that $\Psi=\phi^{-1}$ maps $B$ to $B ; \phi$ is thus an analytic automorphism of $B$, and a theorem due to Harris [Ha], the so-called analytic Banach-Stone Theorem shows that the analogue of (40) holds, namely

$$
\phi(z)=\left(\varepsilon_{j} \frac{z_{\sigma(j)}-a_{j}}{1-\bar{a}_{j} z_{\sigma(j)}}\right)_{j \geq 1}
$$

where $\left(a_{j}\right) \in B,\left|\varepsilon_{j}\right|=1$, and $\sigma$ is a permutation of $\mathbb{N}$.

As before, we must have $a_{j}=0$ for each $j$, and finally the assumption $\phi_{k}(z)=z_{k}^{d_{k}} u_{k}(z)$ implies that $\sigma$ is the identity.

The case of isometries goes as follows (see [BFLQ1]:

Theorem 5.6. Assume that $\phi=\left(\phi_{j}\right): D^{k} \rightarrow \bar{D}^{k}$ induces a composition operator $C_{\phi}$ : $A^{+}\left(\mathbb{T}^{k}\right) \rightarrow A^{+}\left(\mathbb{T}^{k}\right)$. Then, $C_{\phi}$ is an isometry if and only if there exists a square $(k \times k)$ matrix $A=\left(a_{i j}\right)$, with $a_{i j} \in \mathbb{N}_{0}$ and det $A \neq 0$, and complex signs $\varepsilon_{1}, \ldots, \varepsilon_{k}$ such that

$$
\phi_{i}(z)=\varepsilon_{i} z_{1}^{a_{i 1}} \ldots z_{k}^{a_{i k}}, \quad 1 \leq i \leq k, z=\left(z_{1}, \ldots, z_{k}\right) \in D^{k} .
$$

Proof. If $\alpha=\left(\alpha_{1}, \ldots, \alpha_{k}\right) \in \mathbb{N}_{0}^{k}$, let $\phi^{\alpha}=\phi_{1}^{\alpha_{1}} \ldots \phi_{k}^{\alpha_{k}}$. If $f(z)=\sum a_{\alpha} z^{\alpha} \in A^{+}\left(\mathbb{T}^{\infty}\right)$, we write $a_{\alpha}=\hat{f}(\alpha)$ and denote by $\operatorname{Sp} f$ (the spectrum of $f$ ) the set of $\alpha$ 's for which $\hat{f}(\alpha) \neq 0$. One has the following facts:

FACT 1. $C_{\phi}$ is an isometry if and only if:

a) $\phi_{i}=\varepsilon_{i} F_{i}, 1 \leq i \leq k$, where $\left|\varepsilon_{i}\right|=1, \hat{F}_{i} \geq 0$, and $F_{i}(e)=1=\left\|F_{i}\right\|_{\infty}$, with $e=(1,1, \ldots, 1)$;

b) if $\alpha, \alpha^{\prime} \in \mathbb{N}_{0}^{k}$ are distinct, the spectra of $\phi^{\alpha}$ and $\phi^{\alpha^{\prime}}$ do not intersect.

FACT 2. if $\phi=\left(\phi_{i}\right)$ and if one of the $\phi_{i}$ 's is not a monomial, we can find distinct elements $\alpha, \alpha^{\prime} \in \mathbb{N}_{0}^{k}$ such that the spectra of $\phi^{\alpha}$ and $\phi^{\alpha^{\prime}}$ intersect.

Fact 2 needs some arithmetical discussion, but once we have these facts, Theorem 5.6 is clear.

In the case of $A^{+}\left(\mathbb{T}^{\infty}\right)$, we have no such pleasant statement: for example, if $I_{1}, \ldots, I_{m}, \ldots$ are disjoint subsets of $\mathbb{N}$ with more than one element, $c_{i j}$ positive numbers such that $\sum_{j \in I_{i}} c_{i j}=1$ for each $i \geq 1$, and if the map $\phi=\left(\phi_{i}\right)$ is defined by 
$\phi_{i}(z)=\sum_{j \in I_{i}} c_{i j} z_{j}$, then $C_{\phi}$ is an isometry by Fact 1 (which holds for $A^{+}\left(\mathbb{T}^{\infty}\right)$ ), and yet no $\phi_{i}$ is a monomial. Under the additional assumption that $\phi$ maps $\mathbb{T}^{\infty}$ to itself, we have a more satisfactory statement, namely

THEOREM 5.7. Let $\phi: \bar{D}^{\infty} \rightarrow \bar{D}^{\infty}$ a map inducing a composition operator $C_{\phi}: A^{+}\left(\mathbb{T}^{\infty}\right)$ $\rightarrow A^{+}\left(\mathbb{T}^{\infty}\right)$, and such that moreover $\phi\left(\mathbb{T}^{\infty}\right) \subset \mathbb{T}^{\infty}$. Then:

a) There exists a matrix $A=\left(a_{i j}\right)_{i, j \geq 1}$, with $a_{i j} \in \mathbb{N}_{0}$ and $\sum_{j} a_{i j}<\infty$ for each $i$, and complex signs $\varepsilon_{i}$ such that $\phi=\left(\phi_{i}\right)$ and

$$
\phi_{i}(z)=\varepsilon_{i} \prod_{j=1}^{\infty} z_{j}^{a_{i j}}, \quad i=1,2, \ldots
$$

b) $C_{\phi}$ is an isometry if and only if $A^{*}=\left(a_{j i}\right)$ is injective on $\mathbb{Z}^{(\infty)}$.

The main point is the following group-theoretical property [R].

THEOREM 5.8 (Beurling-Helson). Let $G$ be a discrete abelian group, with connected dual group $\Gamma$. Let $\phi \in A(\Gamma)$, the Wiener algebra of $\Gamma$, without zeros on $\Gamma$, and such that $\left\|\phi^{n}\right\|_{A(\Gamma)} \leq C$ for some constant $C=(n=0, \pm 1, \pm 2, \ldots)$. Then, $\phi$ is affine, i.e. there exists a complex number a with $|a|=1$ and an element $x$ of $G$ such that $\phi(\gamma)=a \gamma(x)$ for any $\gamma \in \Gamma$.

Recall that $A(\Gamma)=\left\{\phi: \Gamma \rightarrow \mathbb{C}: \phi(\gamma)=\sum_{1}^{\infty} a_{n} \gamma\left(x_{n}\right)\right.$, with $x_{n} \in G$ and $\sum_{1}^{\infty}\left|a_{n}\right|$ $<\infty\}$; we set $\|\phi\|_{A(\Gamma)}=\sum_{1}^{\infty}\left|a_{n}\right|$. What the lemma says is the following: take for example $G=\mathbb{Z}, \Gamma=\mathbb{T}, \phi \in A^{+}(\mathbb{T})$. The assumption $\left\|\phi^{n}\right\| \leq C$ for $n \in \mathbb{N}$ does not say too much on $\phi$, as the example $\phi(z)=\frac{1+z-z^{2}}{\sqrt{5}}$ of Section 2 shows; but the assumption $\left\|\phi^{n}\right\| \leq C$ for $n \in \mathbb{Z}$ says much more; and precisely, if we assume that $\phi \in A^{+}(\mathbb{T})$ satisfies $\left\|\phi^{n}\right\| \leq C$ for $n \in \mathbb{N}$ and $\left|\phi\left(e^{i t}\right)\right|=1$, we automatically have $\left\|\phi^{n}\right\| \leq C$ for $n \in \mathbb{Z}$, since for $n \in \mathbb{N}$ we have $\left\|\phi^{-n}\right\|=\left\|\bar{\phi}^{n}\right\|=\left\|\phi^{n}\right\|$. Now, if we use this lemma with $G=\mathbb{Z}^{(\infty)}$, the direct sum of countably many copies of $\mathbb{Z}$, and $\Gamma=\mathbb{T}^{\infty}$, the complete direct sum of countably many copies of $\mathbb{T}$, we get (43).

b) follows easily, letting $A$ and $A^{*}$ act on $\mathbb{Z}^{(\infty)}$ by the formulas $A(\alpha)=B, A^{*}(\alpha)=\gamma$, with $\beta_{i}=\sum_{j} a_{i j} \alpha_{j}, \gamma_{j}=\sum_{i} a_{i j} \alpha_{i}$.

We then have, if $C_{\phi}$ is an isometry: $C_{\phi}\left(z^{\alpha}\right)=\phi^{\alpha}=\varepsilon^{\alpha} z^{A^{*}(\alpha)}$, and we know from the proof of Theorem 5.6 that the $\phi^{\alpha}$ 's have disjoint supports, therefore the $A^{*}(\alpha)$ 's are distinct.

If we combine both properties (automorphism, isometry), we get the following (recall that $B$ is the open unit ball of $\left.c_{0}\right)$.

THEOREM 5.9. Let $\phi=\left(\phi_{j}\right): B \rightarrow B$ be an analytic function which induces a composition operator $C_{\phi}$ on $A^{+}\left(\mathbb{T}^{\infty}\right)$. If $C_{\phi}$ is an isometric automorphism of $A^{+}\left(\mathbb{T}^{\infty}\right)$, then $\phi(z)=\left(\varepsilon_{j} z_{\sigma(j)}\right)_{j}$, for some permutation $\sigma$ of $\mathbb{N}$ and some sequence $\left(\varepsilon_{j}\right)_{j}$ of complex signs.

This follows from an inspection of the proof of Theorem 5.5; if $\Psi=\phi^{-1}$ does not map $B$ to $B$, we find distinct integers $j_{1}, j_{2}$ such that the spectra of $\phi_{j_{1}}$ and $\phi_{j_{2}}$ intersect, which prevents $C_{\phi}$ from being isometric. 
Now, we return to the case of the Wiener-Dirichlet algebra $\mathcal{A}^{+}$, for which the statements are more satisfactory than for $A^{+}\left(\mathbb{T}^{\infty}\right)$.

Theorem 5.10. Let $C_{\phi}: \mathcal{A}^{+} \rightarrow \mathcal{A}^{+}$be a composition operator. The following are equivalent:

a) $\phi$ is a vertical translation: $\phi(s)=s+i \tau, \tau \in \mathbb{R}$.

b) $\phi$ is a automorphism.

Proof. b) $\Rightarrow$ a). We write $\phi(s)=c_{0} s+\varphi(s) . C_{\phi}$ being surjective, $\phi$ is injective, and $c_{0} \geq 1$, by a well-known result [Fa] of the theory of analytic, almost-periodic functions. We will now use the transfer operator $\Delta$ introduced at the beginning of Section 5 , by setting $f_{k}(s)=p_{k}^{-\phi(s)} \in \mathcal{A}^{+}, \phi_{k}=\Delta f_{k}$ and

$$
\widetilde{\phi}=\left(\phi_{1}, \phi_{2}, \ldots\right) \text {. }
$$

We have from (33): $\widetilde{\phi}\left(z^{[s]}\right)=\left(\Delta f_{k}\left(z^{[s]}\right)\right)=\left(f_{k}(s)\right)=z^{[\phi(s)]}$, and $\left\|\phi_{k}\right\|_{\infty}=\left\|f_{k}\right\|_{\infty}$ by (34). Moreover, no $\phi_{k}$ is constant, so the open mapping theorem implies that $\left|\phi_{k}(z)\right|<1$ for $z \in B$, i.e. $\widetilde{\phi}(z) \in D^{\infty}$ for $z \in B$. Let us show that in fact $\widetilde{\phi}(z) \in B$. We have $f_{k}(s)=p_{k}^{-c_{0} s} p_{k}^{-\varphi(s)}=p_{k}^{-c_{0} s} g_{k}(s)$, with $\left\|g_{k}\right\|_{\mathcal{A}^{+}}=\left\|C_{\varphi}\left(p_{k}^{-s}\right)\right\|_{\mathcal{A}^{+}} \leq C$. So that $\phi_{k}(z)=$ $z_{k}^{c_{0}} \Delta g_{k}(z)$, and by $(34)$,

$$
\left|\phi_{k}(z)\right| \leq\left|z_{k}\right|^{c_{0}}\left\|\Delta g_{k}\right\|_{\infty}=\left|z_{k}\right|^{c_{0}}\left\|g_{k}\right\|_{\infty} \leq\left|z_{k}\right|^{c_{0}}\left\|g_{k}\right\|_{\mathcal{A}^{+}} \leq C\left|z_{k}\right|^{c_{0}} .
$$

Therefore, $\widetilde{\phi}$ maps $B$ to $B$. Moreover, setting $T=\Delta C_{\phi} \Delta^{-1}: A^{+}\left(\mathbb{T}^{\infty}\right) \rightarrow A^{+}\left(\mathbb{T}^{\infty}\right)$, one easily checks that $T$ is nothing but the composition operator $C_{\widetilde{\phi}}$ associated with $\widetilde{\phi}$. For this $\widetilde{\phi}$, we are fortunately in a position to apply Theorem 5.5: indeed we have that $T$ is an automorphism and that

$$
\phi_{k}(z)=z_{k}^{c_{0}} \Delta g_{k}(z)
$$

with

$$
\Delta g_{k}(0)=\lim _{\Re s \rightarrow \infty} p_{k}^{-\varphi(s)}=p_{k}^{-c_{1}} \neq 0,
$$

and we know that $c_{0} \geq 1$.

Theorem 5.5 now implies that $\widetilde{\phi}(z)=\left(\varepsilon_{j} z_{j}\right)_{j}$, for some sequence $\left(\varepsilon_{j}\right)$ of complex signs. If we test this equality at the points $z^{[s]}=\left(p_{j}^{-s}\right)_{j}, s \in \mathbb{C}_{0}$, and if we use (33), we get

$$
p_{j}^{-\phi(s)}=\varepsilon_{j} p_{j}^{-s}, \quad s \in \mathbb{C}_{0}, j \in \mathbb{N} .
$$

Taking the moduli, we obtain $\Re \phi(s)=\Re s$. Since $\phi(s)-s$ is analytic in the domain $\mathbb{C}_{0}$ this implies $\phi(s)-s=i \tau$, with $\tau \in \mathbb{R}$.

Theorem 5.11. Let $C_{\phi}: \mathcal{A}^{+} \rightarrow \mathcal{A}^{+}$be a composition operator. The following are equivalent:

a) $\phi(s)=c_{0} s+i \tau$, with $c_{0} \in \mathbb{N}$ and $\tau \in \mathbb{R}$.

b) $C_{\phi}$ is an isometry.

Proof. b) $\Rightarrow$ a). Here, we do not need the transfer operator $\Delta$, but clearly the method of proof of Theorem 5.5 works to show that

(45) If $m$ and $n$ are distinct integers, the spectra of $m^{-\phi}$ and $n^{-\phi}$ are disjoint. 
This automatically implies $c_{0} \neq 0$, otherwise $1 \in S p\left(n^{-\phi}\right)$ for each $n$. Now if $\phi(s)=$ $c_{0} s+c_{1}+\omega(s)$, with $\omega(s)=c_{r} r^{-s}+c_{r+1}(r+1)^{-s}+\ldots, r \geq 2$ and $c_{r} \neq 0$, one easily checks that $(n r)^{c_{0}} \in S p\left(n^{-\phi}\right) \cap S p(n r)^{-\phi}$ for large $n$, contradicting (45). Therefore, $\omega=0$ and we are done.

The results of this section are mainly taken from [BFLQ1].

6. Concluding remarks and questions. 1) Let $\phi=\left(\phi_{1}, \ldots, \phi_{k}\right): \bar{D}^{k} \rightarrow \bar{D}^{k}$, nonconstant, with $C_{\phi}: A^{+}\left(\mathbb{T}^{k}\right) \rightarrow A^{+}\left(\mathbb{T}^{k}\right)$. If $k=1$, we must have $\phi(D) \subset D$, and this is used implicitly in the proof of Theorem 2.2 ; but if $k>1$, some component of $\phi$ (e.g. $\left.\phi\left(z_{1}, z_{2}\right)=\left(\frac{1}{2} z_{1}, 1\right)\right)$ might be a unimodular constant, and the analogue of Theorem 2.2 is (46) If $\phi=\left(\phi_{1}, \ldots, \phi_{k}\right): \bar{D}^{k} \rightarrow \bar{D}^{k}$ induces a bounded operator $C_{\phi}$ on $A^{+}\left(\mathbb{T}^{k}\right)$, $C_{\phi}$ is compact if and only if each component $\phi_{j}$ is either constant or such that $\left\|\phi_{j}\right\|_{\infty}<1$.

2) Our knowledge of compact, or even Hilbert-Schmidt, composition operators $C_{\phi}$ on $\mathcal{H}^{2}$ is far from being satisfactory. Bayart [B2] proved the following:

Theorem 6.1. Let $C_{\phi}: \mathcal{H}^{2} \rightarrow \mathcal{H}^{2}$ be bounded. Then:

a) If $C_{\phi}$ is Hilbert-Schmidt, we must have $\phi\left(\mathbb{C}_{0}\right) \subset \mathbb{C}_{\frac{1}{2}}$.

b) If $\phi\left(\mathbb{C}_{0}\right) \subset \mathbb{C}_{\frac{1}{2}+\varepsilon}$ for some $\varepsilon>0$, then $C_{\phi}$ is Hilbert-Schmidt.

We have already seen in Theorem 4.11 that the assumption of b) is not necessary, although it is necessary (see Theorems $4.9,4.10$ ) for symbols $\phi(s)=c_{0} s+c_{1}+\sum_{j=1}^{d} c_{q_{j}} q_{j}^{-s}$, where $q_{1}, \ldots, q_{d}$ are independent and $d=1$ or 2 .

Using the properties of Hermite polynomials (see Section 5), we were able to prove [BFLQ2]:

THEOREM 6.2. Let $r \in \mathbb{N}, r \geq 2$, and $\phi(s)=c_{0} s+c_{1}+c_{r} r^{-s}+c_{r^{2}} r^{-2 s}$, with $c_{r}, c_{r^{2}}>0$. Then, the following are equivalent:

a) $C_{\phi}: \mathcal{H}^{2} \rightarrow \mathcal{H}^{2}$ is Hilbert-Schmidt.

b) $\phi\left(\mathbb{C}_{0}\right) \subset \mathbb{C}_{\frac{1}{2}+\varepsilon}$ for some $\varepsilon>0$.

3) An irritating question is the following: if $\phi(s)=c_{0} s+\varphi(s)$, with $\varphi \in \mathcal{D}$, and if we know that $C_{\phi}: \mathcal{A}^{+} \rightarrow \mathcal{A}^{+}$, is it true that $\varphi \in \mathcal{A}^{+}$?

Theorem 5.3 might indicate that this is not so; on the other hand, it would be interesting, in this Theorem, to treat the case of complex coefficients $c_{r}, c_{r^{2}}$. Here, recent estimates due to Rusev [Ru] might help.

4) The estimate $\left\|\phi^{n}\right\|_{A^{+}} \geq \delta \sqrt{n}$ of Lemma 2.4 is best possible. In fact (see [4], p. 76), it is fairly easy to see that $\left\|\phi^{n}\right\|_{A^{+}} \leq C \sqrt{n}$ if $\phi=e^{i g}$ and $g$ is $C^{\infty}$ (say), and a similar computation in dimension $k$ (i.e. if we work with $A^{+}\left(\mathbb{T}^{k}\right)$ ) easily gives the estimate $\left\|\phi^{n}\right\|_{A^{+}\left(\mathbb{T}^{k}\right)} \leq C_{k} n^{k / 2}$. Conversely, if $\phi$ is not affine, we probably have $\left\|\phi^{n}\right\|_{A^{+}\left(\mathbb{T}^{k}\right)} \geq \delta \sqrt{n}$. When do we have the "best" minorization $\left\|\phi^{n}\right\|_{A^{+}\left(\mathbb{T}^{k}\right)} \geq \delta n^{k / 2}$ ? In general, it would be interesting to have a precise quantitative version of Beurling-Helson's lemma 5.8 when $\phi$ is not affine.

5) In the proof of Theorem 5.10, we used the fact that an analytic almost-periodic function $\sum a_{n} e^{-\lambda_{n} s}$, uniformly convergent in a strip $a<\Re s<b$, is never injective on 
this strip. Now, can an almost-periodic function $f: \mathbb{R} \rightarrow \mathbb{C}$ be injective? (Of course, if $f$ is real-valued, this is impossible: if $f$ is injective, it is monotonic, therefore not almost-periodic).

6) The ugly Theorem 5.5 is yet sufficient to describe the composition automorphisms of $\mathcal{A}^{+}$(observe in passing that Bayart's direct approach for $\mathcal{H}^{2}$ does not seem to work here, which explains our "detour" through $\left.A^{+}\left(\mathbb{T}^{\infty}\right)\right)$. Is the natural statement true, i.e. is it true that $C_{\phi}$ is an automorphism of $A^{+}\left(\mathbb{T}^{\infty}\right)$ if and only if $\phi(z)=\left(\varepsilon_{j} z_{\sigma(j)}\right)$ for some permutation $\sigma$ of $\mathbb{N}$ and some sequence $\left(\varepsilon_{j}\right)$ of complex signs?

7 ) It is not true in general that the composition operator $C_{\widetilde{\phi}}$ on $A^{+}\left(\mathbb{T}^{\infty}\right)$ corresponds to a composition operator $C_{\phi}$ on $\mathcal{A}^{+}$, in (44) of the proof of Theorem 5.10; for example, if $\widetilde{\phi}=\left(\phi_{i}\right)$, with

$$
\phi_{i}(z)=\frac{z_{2 i-1}+z_{2 i}}{2}, \quad i=1,2, \ldots,
$$

the equation $\widetilde{\phi}\left(z^{[s]}\right)=z^{[\phi(s)]}$ would give

$$
\frac{p_{2 i-1}^{-s}+p_{2 i}^{-s}}{2}=p_{i}^{-\phi(s)}, \quad i=1,2, \ldots ;
$$

taking equivalents of both members as $s \rightarrow \infty$ would give that

$$
\frac{\phi(s)}{s} \rightarrow \frac{\log p_{2 i-1}}{\log p_{2 i}}
$$

and it is impossible to have that, even for one $i$, since $\frac{\phi(s)}{s} \rightarrow c_{0} \in \mathbb{N}_{0}$ !

Acknowledgments. The author thanks J. Zemánek and Y. Tomilov for giving him the opportunity to come to the Workshop on Operator Theory in Warsaw in April 2004, and to write this survey.

\section{References}

[Al] G. Alexits, Convergence Problems of Orthogonal Series, Pergamon Press, 1961.

[Ar] W. Arveson, A Short Course on Spectral Theory, Springer-Verlag 2002.

[B1] F. Bayart, Hardy spaces of Dirichlet series and their composition operators, Monat. Math. 136 (2002), 203-236.

[B2] F. Bayart, Compact composition operators on a Hilbert space of Dirichlet series, Illinois J. Math. 47 (2003), 725-743.

[B3] F. Bayart, Opérateurs de composition sur les espaces de séries de Dirichlet et problèmes d'hypercyclicité simultanée, Thèse de l'Université de Lille 1, 2002.

[BFLQ1] F. Bayart, C. Finet and D. Li and H. Queffélec, Composition operators on the Wiener-Dirichlet algebra, J. Oper. Theory, to appear.

[BFLQ2] F. Bayart, C. Finet, D. Li and H. Queffélec, New results on composition operators in the Dirichlet series setting, in preparation.

[Bo] H. Bohr, Über die gleichmässige Konvergenz Dirichletscher Reihen, J. Reine Angewandte Math. 143 (1913), 203-211.

[Co] C. C. Cowen, Composition operators on $H^{2}$, J. Oper. Theory 9 (1983), 77-106.

$[\mathrm{CoMcCl}] \quad$ C. C. Cowen and B. MacCluer, Composition Operators on Spaces of Analytic Functions, CRC Press, Boca Raton, 1995. 
[Fa] J. Favard, Leçons sur les fonctions presque-périodiques, Gauthier-Villars, 1933.

[Fe] H. Federer, Geometric Measure Theory, Springer-Verlag, 1969.

[FQ] C. Finet and H. Queffélec, Numerical range of composition operators on a Hilbert space of Dirichlet series, Linear Algebra and its Applications 377 (2004), 1-10.

[FQV] C. Finet, H. Queffélec and A. Volberg, Compactness of composition operators on a Hilbert space of Dirichlet series, J. Funct. Analysis 211 (2004), 271-287.

[GH] J. Gordon and H. Hedenmalm, The composition operators on the space of Dirichlet series with square-summable coefficients, Michigan Math. J. 46 (1999), 313-329.

[Ha] L. A. Harris, Schwarz's lemma in normed linear spaces, Proc. Acad. Sci. USA 62 (1969), 1014-1017.

[HLS] H. Hedenmalm, P. Lindqvist and K. Seip, A Hilbert space of Dirichlet series and a system of dilated function in $L^{2}(0,1)$, Duke Math. J. 86 (1997), 1-36.

[He] H. Helson, Compact groups and Dirichlet series, Ark. Mat. 8 (1969), 139-143.

[HJ] H. Hunziker and H. Jarchow, Composition operators which improve integrability, Math. Nachr. 152 (1991), 83-99.

[J] N. Jaoua, Similarity to a contraction and hypercontractivity of composition operators, Proc. Amer. Math. Soc. 129 (2001), 2085-2092.

[Kal] J.-P. Kahane, Séries de Fourier absolument convergentes, Springer-Verlag, 1970.

[Ka2] J.-P. Kahane, Some Random Series of Functions, Cambridge University Press, 1985.

[LiQ] D. Li and H. Queffélec, Introduction à l'étude des espaces de Banach, Analyse et Probabilités, Cours spécialisé de la SMF no. 12, 2004.

[McCl] B. MacCluer, Fredholm composition operators, Proc. Amer. Math. Soc. 125 (1997), 163-166.

[McCa] J. E. McCarthy, Hilbert spaces of Dirichlet series and their multipliers, Trans. Amer. Math. Soc. 356 (2004), 881-893.

[McClS] B. D. MacCluer and J. Shapiro, Angular derivatives and compact composition operators on the Hardy and Bergman spaces, Canadian J. Math. 38 (1986), 878-906.

[MVau H. L. Montgomery and R. C. Vaughan, Hilbert's inequality, J. London Math. Soc. (2) 8 (1974), 73-82.

[MVaa] H. L. Montgomery and J. D. Vaaler, A further generalization of Hilbert's inequality, Mathematika 45 (1998), 35-39.

[Na] R. Narasimhan, Several Complex Variables, Chicago Lectures in Math., 1971.

[Ne] D. J. Newman, Homomorphisms of $\ell_{+}$, American J. Math. 91 (1969), 37-46.

[No] E. A. Nordgren, Composition operators, Canadian J. Math. 20 (1968), 442-449.

[P] J. Peyrière, personal communication.

[Q1] H. Queffélec, Propriétés presque sûres et quasi-sûres des séries de Dirichlet et des produits d'Euler, Canad. J. Math. 32 (1980), 531-558.

[Q2] H. Queffélec, Harald Bohr's vision of Dirichlet series: Old and new results, J. Analysis 3 (1995), 43-60.

[Re] A. Rényi, Calcul des Probabilités, Dunod, 1966.

[R] W. Rudin, Fourier Analysis on Groups, Interscience Publishers, 1962.

[Ru] P. Rusev, An inequality for Hermite's polynomials in the complex plane, C. R. Acad. Bulgare Sci. 53 (2000), no. 10, 13-16.

[Sc] H. J. Schwartz, Composition operators on $H^{p}$, Thesis, Univ. of Toledo, 1968.

[S1] J. Shapiro, The essential norm of a composition operator, Annals of Math. 125 (1987), 375-404.

[S2] J. Shapiro, Composition Operators and Classical Function Theory, Springer-Verlag, 1991. 
